\title{
Perpendicular magnetic anisotropy in granular multilayers of CoPd alloyed nanoparticles
}

\author{
L. G. Vivas \\ Instituto de Ciencia de Materiales de Aragón, CSIC - Universidad de Zaragoza. Dept. Física de la Materia Condensada, \\ E-50009 Zaragoza, Spain \\ J. Rubín* \\ Instituto de Ciencia de Materiales de Aragón, CSIC - Universidad de Zaragoza. Dept. Ciencia y Tecnología de Materiales y Fluidos, \\ E-50018 Zaragoza, Spain
}

A. I. Figueroa, F. Bartolomé, and L. M. García

Instituto de Ciencia de Materiales de Aragón, CSIC - Universidad de Zaragoza. Dept. Física de la Materia Condensada, E-50009 Zaragoza, Spain

C. Deranlot and F. Petroff

Unité Mixte de Physique CNRS/Thales, F-91767 Palaiseau Cedex, France

and Université Paris-Sud, F-91405 Orsay Cedex, France

L. Ruiz and J. M. González-Calbet

Dept. Química Inorgánica, Universidad Complutense de Madrid, E-28040 Madrid, Spain

S. Pascarelli, N. B. Brookes, F. Wilhelm, M. Chorro, and A. Rogalev

European Synchrotron Radiation Facility CS40220, F-38043 Grenoble Cedex 9, France

J. Bartolomé

Instituto de Ciencia de Materiales de Aragón, CSIC - Universidad de Zaragoza. Dept. Física de la Materia Condensada, E-50009 Zaragoza, Spain

(Received 27 January 2016; revised manuscript received 28 March 2016; published 16 May 2016)

\begin{abstract}
Co-Pd multilayers obtained by Pd capping of pre-deposited Co nanoparticles on amorphous alumina are systematically studied by means of high-resolution transmission electron microscopy, $\mathrm{x}$-ray diffraction, extended $\mathrm{x}$-ray absorption fine structure, SQUID-based magnetometry, and $\mathrm{x}$-ray magnetic circular dichroism. The films are formed by CoPd alloyed nanoparticles self-organized across the layers, with the interspace between the nanoparticles filled by the non-alloyed Pd metal. The nanoparticles show atomic arrangements compatible with short-range chemical order of $\mathrm{L}_{0}$ strucure type. The collective magnetic behavior is that of ferromagnetically coupled particles with perpendicular magnetic anisotropy, irrespective of the amount of deposited Pd. For increasing temperature three magnetic phases are identified: hard ferromagnetic with strong coercive field, soft-ferromagnetic as in an amorphous asperomagnet, and superparamagnetic. Increasing the amount of Pd in the system leads to both magnetic hardness increment and higher transition temperatures. Magnetic total moments of 1.77(4) $\mu_{B}$ and 0.45(4) $\mu_{B}$ are found at Co and Pd sites, respectively, where the orbital moment of Co, 0.40(2) $\mu_{B}$, is high, while that of $\mathrm{Pd}$ is negligible. The effective magnetic anisotropy is the largest in the capping metal series ( $\mathrm{Pd}, \mathrm{Pt}, \mathrm{W}, \mathrm{Cu}, \mathrm{Ag}, \mathrm{Au})$, which is attributed to the interparticle interaction between de nanoparticles, in addition to the intraparticle anisotropy arising from hybridization between the $3 d-4 d$ bands associated to the Co and Pd chemical arrangement in a $\mathrm{L1}_{0}$ structure type.
\end{abstract}

DOI: 10.1103/PhysRevB.93.174410

\section{INTRODUCTION}

The research on the interplay between magnetic and structural properties at the nanoscale is essential for the control of the magnetic anisotropy, the understanding of new size-effect induced physical phenomena, and for technological purposes, as in products based on new functional magnetic materials [1,2] and magnetic nanoparticle-based cancer treatments $[3,4]$. In particular, extensive works have been driven towards the ability to stabilize a magnetic easy axis perpendic-

*jrubin@unizar.es ular to the substrate plane, which is central to magneto-optical storage and perpendicular magnetic media [5-7]. In this context, the research on bimetallic magnetic nanoparticles (NPs) of $3 d$ ferromagnets and heavy metals has attracted much attention as they offer new possibilities to tune the material performance aiming to control the magnetic anisotropy energy (MAE) [8,9].

$\mathrm{Co} / \mathrm{M}$ multilayer systems have been studied for about 10 transition metals including $3 d, 4 d$ and $5 d$ elements (see Ref. [10] for a review). In particular, $\mathrm{Co}_{1-x} \mathrm{Pd}_{x}$ alloy films and $\mathrm{Co} / \mathrm{Pd}$ multilayers show perpendicular magnetic anisotropy (PMA) and they have been extensively studied in order to understand the origin of its PMA. PMA has been reported 
in a variety of combinations of $\mathrm{Co}$ and $\mathrm{Pd}$ in the form of thin film alloys [5,11,12] and thin film multilayers [13]. The origin of PMA in $\mathrm{Co} / \mathrm{Pd}$ multilayers has been attributed mainly to magnetic interface anisotropy [14], while in Co-Pd film alloys, PMA has been explained by both anisotropic strain and short range ordering of the Co atoms in the Pd matrix [15]. In contrast, Co-Pd alloy films in disordered phase are not expected to exhibit PMA [12]. More complexity arises at small thicknesses, when Co and Pd aggregate as alloyed NPs and additional sources of anisotropy should be included.

As in all-metallic Co/Pd multilayer systems [16], interlayer coupling could also be observed in CoPd agregates laid as multilayers under certain spacers and conditions. However, interlayer coupling can be avoided by introducing non magnetic isolating spacers like $\mathrm{Al}_{2} \mathrm{O}_{3}$, which allows for a direct study of the interactions within the magnetic layers. For Co nominal thickness above $t_{\mathrm{Co}}=0.6 \mathrm{~nm}$ deposited on $\mathrm{Al}_{2} \mathrm{O}_{3}$, Co nanoparticles aggregate up to a percolation threshold of $\approx 2 \mathrm{~nm}[17,18]$. The systems with $t_{\mathrm{Co}}=0.7 \mathrm{~nm}$, where a non-magnetic transition metal is subsequently deposited, could be used to study magnetic ordering and anisotropy induced by a metallic spacer connecting magnetic NPs within the Co-Pd layer. In previous works our group has studied $\left[\mathrm{Al}_{2} \mathrm{O}_{3} / \mathrm{Co} / \mathrm{M}\right]_{N}$ multilayers made of layers of Co NPs capped with different nonmagnetic transition metals $\mathrm{M}=\mathrm{Au}, \mathrm{Ag}, \mathrm{Cu}, \mathrm{W}, \mathrm{Pt}$, and separated by thin amorphous $3 \mathrm{~nm}$-thick $\mathrm{Al}_{2} \mathrm{O}_{3}$ layers [19-21]. Capping the Co NPs with metals which do not diffuse into $\mathrm{Co}$, like the noble metals $\mathrm{Au}$ and $\mathrm{Ag}$, or $\mathrm{Cu}$, results in an increase in the surface anisotropy of the Co NPs $[19,20]$. In contrast, capping with the nonmagnetic elements $\mathrm{W}$ or $\mathrm{Pt}$, which do alloy with $\mathrm{Co}$, modifies the intrinsic magnetic anisotropy. Indeed, while the Co-W NPs are amorphous, albeit magnetically anisotropic [22], the Co-Pt alloyed NPs present PMA and an atomically ordered structure with short range $\mathrm{L} 1_{0}$ arrangement [21], which has been suggested as the origin of the observed PMA. Moreover, the Pt multilayer samples constitute the only case of all those mentioned above which shows ferromagnetic order within the layers.

The onset of magnetic order in the platinum system leads to extend the study of the $\mathrm{Al}_{2} \mathrm{O}_{3} / \mathrm{Co} / \mathrm{M}$ multilayers to palladium as a natural next step, where the specific modifications introduced by the $4 d$ element Pd can be compared to the close $5 d$ element Pt. The case of Pd introduces two opposite effects with respect to that of Pt. On one hand, its spin-orbit coupling is about 3 times smaller $\left(\xi_{\mathrm{Pd}}=195 \mathrm{meV}, \xi_{\mathrm{Pt}}=600 \mathrm{meV}\right)$ [23], which should lead to a lower magnetic anisotropy; this could be partially compensated by the larger Pauli and orbital paramagnetic components found for the magnetic susceptibility in $\mathrm{Pd}$ metal than in $\mathrm{Pt}$ [24]. On the other hand, the $\mathrm{Pd} 4 d$ electronic band is closer in energy to the Co $3 d$ band, which could favour stronger hybridization under appropriate atomic arrangements. In fact, the degree of alloying with $\mathrm{Co}$ and the presence of chemically CoPd ordered phases, like the $\mathrm{L}_{0}$ structure, are expected to play important roles, and calculations made in CoPt and CoPd multilayers with that atomic arrangement two decades ago have shown that the magnetic anisotropy in the case of Pt should be larger [25]. Revealing the actual effect of the aforementioned electronic characteristics of the elements constituting the nanoparticles on the magnetic properties of the multilayer systems requires a close comparison of the
$\mathrm{Al}_{2} \mathrm{O}_{3} / \mathrm{Co} / \mathrm{Pd}$ and $\mathrm{Al}_{2} \mathrm{O}_{3} / \mathrm{Co} / \mathrm{Pt}$ multilayers. To this end, we prepared $\mathrm{Al}_{2} \mathrm{O}_{3} / \mathrm{Co} / \mathrm{Pd}$ multilayers with the same $\mathrm{Co}$ and $\mathrm{Pd}$ thicknesses as in the previously investigated $\mathrm{Pt}$ case and performed a similar experimental study of the structural, morphological, and magnetic properties.

In what follows it will be shown that CoPd nanoparticles are formed with a composition close to $1: 1$ and a Co core smaller than in the Pt counterpart; the magnetic anisotropy is perpendicular to the layer's plane and larger than that of the CoPt nanoparticles, in contrast to predictions, and ferromagnetic order is observed at temperatures higher than in the $\mathrm{Al}_{2} \mathrm{O}_{3} / \mathrm{Co} / \mathrm{Pt}$ multilayers. Moreover, we will show that $\mathrm{Pd}$ presents enhanced spin moment, higher than in $\mathrm{Pt}$, and notice that ferromagnetic order is only observed when the metal capping shows large enhancement, in contrast to the $\mathrm{Cu}$, $\mathrm{Ag}$, Au and W capping metals, where the enhancement is small and there is no magnetic ordering. The results of magnetic anisotropy will be compared to previous calculations of other authors, from which anisotropy can be related to the short range $\mathrm{L} 1_{0}$ chemical order, and we will propose a contribution to magnetic anisotropy of relativistic RKKY type associated to the ferromagnetic order.

The present report is organized as follows. The description of the $\left[\mathrm{Al}_{2} \mathrm{O}_{3} / \mathrm{Co} / \mathrm{Pd}\right]_{N}$ multilayer samples studied and the characterization techniques used is presented in Sec. II. Then, the morphological properties of the multilayers studied by high-resolution transmission electron microscopy (HRTEM) and high-angle annular dark field (HAADF) images will be described in Sec. III. The structural properties are studied in the same section by X-ray diffraction (XRD) and extended $\mathrm{X}$-ray absorption fine structure (EXAFS) analysis. In Sec. IV the magnetic properties derived from SQUID magnetometry and X-ray magnetic circular dichroism (XMCD) measurements at both $\mathrm{Co}$ and $\operatorname{Pd} L_{2,3}$ and $\mathrm{Co} K$ edges are presented and compared to the Pt counterpart. Finally, Sec. V presents the discussion of the results, including a comparison of XMCD results in the Co $K$ edge with our own calculations using the FDMNES code [26,27].

\section{SAMPLE PREPARATION AND EXPERIMENTAL DETAILS}

The $\mathrm{Al}_{2} \mathrm{O}_{3} / \mathrm{Co} / \mathrm{Pd}$ samples were prepared at the Unité Mixte de Physique CNRS/Thales by sequential sputtering deposition of $\mathrm{Al}_{2} \mathrm{O}_{3}, \mathrm{Co}$, and $\mathrm{Pd}$ on $\mathrm{Si}$ or glass substrates, following the procedure described in previous works on metal capped Co nanoparticles [20]. The alumina, cobalt, and palladium were deposited using Ar plasma, the metals in DC mode, and the insulator at a RF power of $2.2 \mathrm{~W} / \mathrm{cm}^{2}$. The substrate temperature was kept constant at $293 \mathrm{~K}$ and the $\mathrm{Ar}$ pressure was $2 \times 10^{-3}$ Torr. The formation of Co aggregates on the amorphous $\mathrm{Al}_{2} \mathrm{O}_{3}$ is the result of three-dimensional growth because of the different surface energies between them [28]. Aggregation occurs below a certain threshold of the deposited nominal thickness of $\mathrm{Co}, t_{\mathrm{Co}}$, that the layer would have if it were continuous. Not all the Co deposited on the alumina layer aggregates as forming the particles, but there exists a fraction of Co atoms or small clusters dispersed on the matrix. More details about cobalt aggregation on alumina can be found elsewhere [29]. The Co clusters are subsequently capped with 
a Pd layer, for which the nominal thickness $t_{\mathrm{Pd}}$, and then the alumina layer of about $3 \mathrm{~nm}$ is deposited on top of this Co-Pd system. The samples follow the formula [substrate $/(3 \mathrm{~nm}$ $\left.\left.\mathrm{Al}_{2} \mathrm{O}_{3} / t_{\mathrm{Co}} \mathrm{Co} / t_{\mathrm{Pd}} \mathrm{Pd}\right)_{N} / \mathrm{Al}_{2} \mathrm{O}_{3}\right]$, with $N$ being the number that the sequence is repeated in order to obtain a granular multilayer system of Co NPs capped with Pd. We fixed $t_{\text {Co }}=0.7 \mathrm{~nm}$, varying $t_{\mathrm{Pd}}=0.6,1.5,4.5$ and $6.0 \mathrm{~nm}$, with $N=25$ in all cases. We also included a reference sample of noncapped Co NPs (bare) with $t_{\mathrm{Co}}=0.7 \mathrm{~nm}, t_{\mathrm{Al}_{2} \mathrm{O}_{3}}=3 \mathrm{~nm}$, and $N=20$. In what follows we will refer to the $\mathrm{Al}_{2} \mathrm{O}_{3} / \mathrm{Co} / \mathrm{Pd}$ multilayer granular system as "CoPd NPs," since the Co clusters are alloyed with the $\mathrm{Pd}$, as we will show later. Bulk reference samples of $\mathrm{Co}_{3} \mathrm{Pd}$, $\mathrm{CoPd}$ and $\mathrm{CoPd}_{3}$ were prepared by arc melting of appropriate amounts of the constituents. The concentrated alloys were heat treated with a slow decreasing temperature ramp from $1000{ }^{\circ} \mathrm{C}$ to room temperature over a period of 21 days.

The morphology of the granular Co-Pd multilayer samples was studied by HRTEM and HAADF. HRTEM images were taken in cross section configuration, in a JEOL JEM 3000 F Field Emission Microscope fitted with an Oxford LINK electron dispersive spectrometry (EDS) analyser and electron energy loss spectrometry (EELS). The crystalline phases were searched by XRD measurements performed at room temperature using a rotating anode D-Max Rigaku diffractometer. The composition of the Co-Pd phases was identified by both EDS and XRD measurements [30].

XAS spectra were measured at the Co and $\mathrm{Pd} K$ edges using a $\mathrm{Si}(111)$ double-crystal monochromator at the former BM29 beamline (now BM23) of the European Synchrotron Radiation Facility (ESRF). Measurements were performed at $300 \mathrm{~K}$ in fluorescence detection mode with a 13 -element Ge solid state detector with digital signal processing for fluorescence XAS, high energy resolution, and high count rate. $\mathrm{Co}_{3} \mathrm{Pd}, \mathrm{CoPd}$, and $\mathrm{CoPd}_{3}$ alloys and Co film reference samples were also measured at the Co $K$ edge. Besides, a Pd film reference sample was measured at the $\mathrm{Pd} K$ edge.

XMCD measurements at the Co $L_{2,3}$ edges on the CoPd NPs were performed at the ESRF former ID08 beamline (now ID32), with incident angle $\gamma=10^{\circ}$ with respect to the sample surface normal. An APPLE-II undulator and a spherical grating monochromator were used in this case. The degree of circular polarization of the incoming photon beam at these Co $L_{2,3}$ edges was $\sim 100 \%$ and the total electron yield (TEY) detection method was employed. The XMCD signal was obtained by applying a magnetic field of $40 \mathrm{kOe}$ along the $\mathrm{X}$-ray beam direction, at a temperature of $7 \mathrm{~K}$. XMCD measurements at the $\mathrm{Pd} L_{2,3}$ edges were performed at the ESRF ID12 beamline and the detection technique was fluorescence in backscattering geometry. The polarization was around $22 \%$ and $12.6 \%$ at the $\operatorname{Pd} L_{2}$ and $L_{3}$ edges, respectively. A magnetic field of $20 \mathrm{kOe}$ was applied with an incident angle of $75^{\circ}$ with respect to the sample surface normal and the temperature was $12 \mathrm{~K}$. These field and temperature were chosen according to the magnetic properties of the CoPd NPs, so that the system was magnetically saturated under these conditions. XMCD was obtained by difference of XAS spectra measured with opposite helicities of the light at a fixed magnetic field value, and flipping the field in two inverse directions for a fixed light polarization.
Magnetization measurements were performed with a SQUID magnetometer equipped with the high resolution option. Both magnetization as a function of the applied field, $M(H)$, and as a function of temperature, $M(T)$, were measured with the field applied parallel $(\|)$ and perpendicular $(\perp)$ to the substrate plane. The $M(T)$ curves were measured after zero-field cooling (ZFC) and field cooling (FC) the sample. Temperature was varied between $5 \mathrm{~K}$ and $400 \mathrm{~K}$ for different applied field values. Hysteresis loops were measured at several temperatures from 5 to $350 \mathrm{~K}$, under applied fields up to $50 \mathrm{kOe}$.

\section{MORPHOLOGICAL AND STRUCTURAL PROPERTIES}

\section{A. Electron microscopy}

Figures 1 and 2 show HRTEM images for the CoPd NPs samples, from which the granular morphology and thickness of each Co-Pd layer are identified. The thickness of each $\mathrm{Co}-\mathrm{Pd}$ metallic layer with nominal $t_{\mathrm{Co}}=0.7 \mathrm{~nm}$ is irregular for $t_{\mathrm{Pd}}=$ $0.6 \mathrm{~nm}$ and $1.5 \mathrm{~nm}$, but becomes rather regular for $t_{\mathrm{Pd}}=4.5$
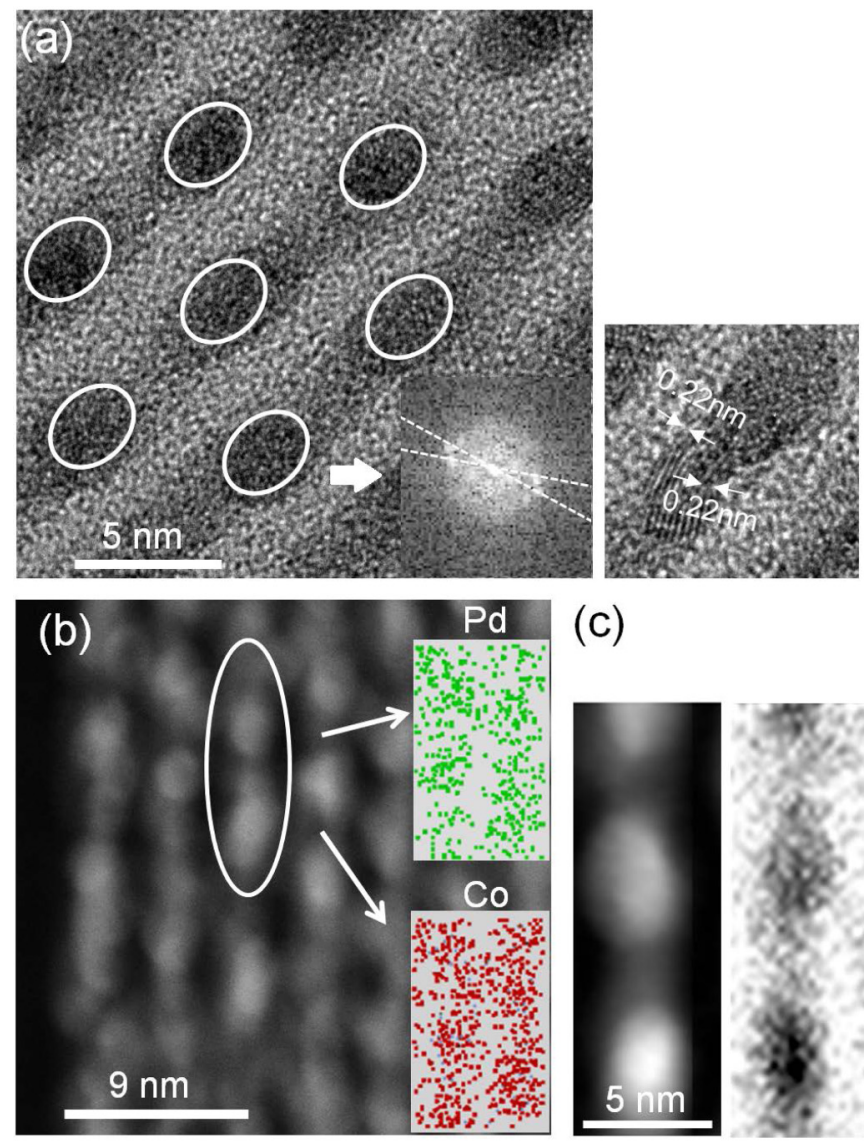

(c)

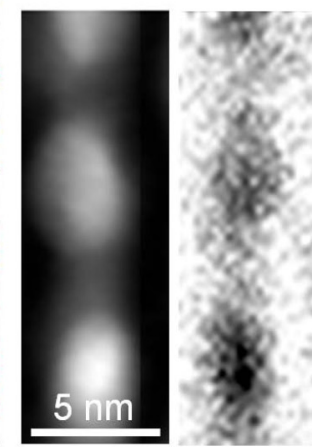

FIG. 1. (a) HRTEM images in cross section configuration for the sample with $t_{\mathrm{Co}}=0.7 \mathrm{~nm}$ and $t_{\mathrm{Pd}}=0.6 \mathrm{~nm}$. (a) The arrangement of the NPs within layers is highlighted. Inset at bottom right corner: a selected zone with its corresponding FFT with the lines indicating (111) planes compatible with $\mathrm{Co}_{x} \mathrm{Pd}_{1-x}$ alloys. Right: interplanar crystalline distances of a selected zone. (b) HAADF image and EDS maps for both $\mathrm{Pd}$ and $\mathrm{Co}$ in the highlighted zone (insets). (c) Zoom of the highlighted zone of the HAADF image in (b) (left) and corresponding EELS Co chemical map (right). 

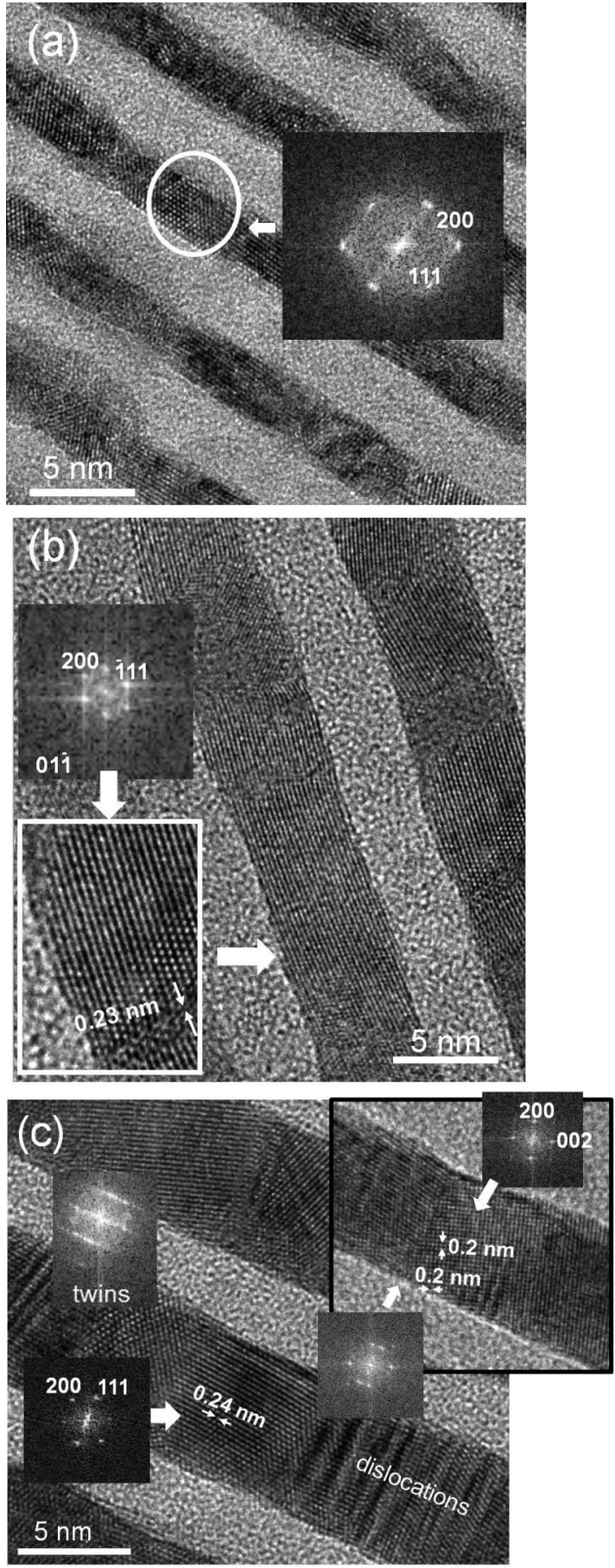

FIG. 2. HRTEM images in cross section configuration for samples with $t_{\mathrm{Co}}=0.7 \mathrm{~nm}$, and (a) $t_{\mathrm{Pd}}=1.5 \mathrm{~nm}$, (b) $t_{\mathrm{Pd}}=4.5 \mathrm{~nm}$ and (c) $t_{\mathrm{Pd}}=6.0 \mathrm{~nm}$. Selected zones with the corresponding FFT are highlighted in all cases, with the spots indexed according to the interplanar distances of an fcc Pd cell. Evidence of twining and dislocations are also highlighted in (c).

and $6.0 \mathrm{~nm}$. The average thickness of the Co-Pd metallic layer is 3.1(2), 3.5(1), 6.3(2) and 7.5(1) nm, respectively. Therefore, in the first two cases $\left(t_{\mathrm{Pd}}=0.6\right.$ and $\left.1.5 \mathrm{~nm}\right)$ it is dominated by the thickness of the Co preformed NPs [17], while in the two latter samples, $\left(t_{\mathrm{Pd}}=4.5\right.$ and $\left.6 \mathrm{~nm}\right)$ their thickness is dominated by the Pd capping. The relation between the actual layer thickness with the nominal value of deposited Pd shows that a uniform Pd capping layer is formed when the free space between nanoparticles is filled. In fact, for Pd deposited thickness above $\sim 1 \mathrm{~nm}$, the layer thickness increases at a rate of $\sim 0.9(1) \mathrm{nm}$ per $\mathrm{nm}$ of $\mathrm{Pd}$.

Figure 1 shows high magnification electron cross-section HRTEM and HAADF images, and EDS and EELS chemical maps of the cross section of the sample with $t_{\mathrm{Co}}=0.7 \mathrm{~nm}$, and $t_{\mathrm{Pd}}=0.6 \mathrm{~nm}$. Light gray layers correspond to the amorphous alumina and darker layers to Co and Pd. The particulates are approximately spherical with diameter $D \sim 3 \mathrm{~nm}$ and are arranged in a hexagonal network within the layers, characteristic of Co deposited on amorphous alumina [20-22]. The fast Fourier transform (FFT) in the nanoparticles shows the existence of crystallized regions with definite crystal planes, alternating with amorphous regions. The high contrast HAADF images and EELS analysis confirm that Co proportion is highest within the particles, but the presence of Pd was confirmed by EDS only. The simultaneous detection of Co and $\mathrm{Pd}$ in the area of the NP proves the formation of alloys, but the relative atomic proportions could not be resolved. Moreover, the EDS maps show that Pd filling of the space between NPs is low in this lowest Pd content multilayer.

HRTEM images for the samples with $t_{\mathrm{Pd}}=1.5,4.5$ and $6.0 \mathrm{~nm}$ are displayed in Fig. 2. Ordered regions alternate with less ordered ones. The local FFT of selected areas of the images shows spots that could be indexed with the interplanar distances of an fcc cell of Pd metal in all cases. The area of well crystallized regions increases with the amount of Pd in the samples, as shown by the enhancement of the intensity and resolution of crystalline spots in the FFT images, when compared to those shown in Fig. 1(a) of the $t_{\mathrm{Pd}}=$ $0.6 \mathrm{~nm}$ sample. The structural and magnetic characterization is presented in the following sections in order to study the alloying between Co and Pd.

\section{B. X-ray diffraction}

$\mathrm{X}$-ray diffraction patterns were collected in the range $20^{\circ}<2 \theta<80^{\circ}$ using $\mathrm{Cu} \mathrm{K} \alpha$ radiation. Figure 3(a) shows the XRD patterns for the $\mathrm{Al}_{2} \mathrm{O}_{3} / \mathrm{Co} / \mathrm{Pd}$ granular films deposited on glass $\left(t_{\mathrm{Pd}}=0.6,4.5\right.$, and $\left.6.0 \mathrm{~nm}\right)$ and on crystalline silicon $\left(t_{\mathrm{Pd}}=1.5 \mathrm{~nm}\right)$, in the range $30^{\circ}<2 \theta<55^{\circ}$, since no reflections were detected out of this range.

A very broad peak is observed at $2 \theta \sim 42^{\circ}$ and $\sim 41^{\circ}$ for the samples with $t_{\mathrm{Pd}}=0.6$ and $1.5 \mathrm{~nm}$, respectively. The shift towards lower angle value in the $t_{\mathrm{Pd}}=1.5 \mathrm{~nm}$ sample is due to the contribution of the (111) Pd reflection. Indeed, as more Pd is deposited $\left(t_{\mathrm{Pd}}=4.5\right.$ and $\left.6 \mathrm{~nm}\right)$, the well defined Pd (111) reflection, with increasing intensity, is located at $2 \theta \sim 40^{\circ}$ followed by a shoulder at $2 \theta \sim 42^{\circ}$. Additionally, there is intensity in the $45^{\circ}$ to $50^{\circ}$ range in all the samples, which is most evident in those samples with the lowest Pd content and is not correlated to the large growth of the (111) Pd reflection peak as $t_{\mathrm{Pd}}$ is deposited. Therefore this part of the diffraction pattern is mainly related to phases formed at the beginning of the Pd deposition.

The diffraction profile was fitted with XRFIT included in the FULLFROF code, using pseudo-Voigt peaks [31]. Up to four peaks were included to fit the intensity in the range $38^{\circ}$ to 
(a)
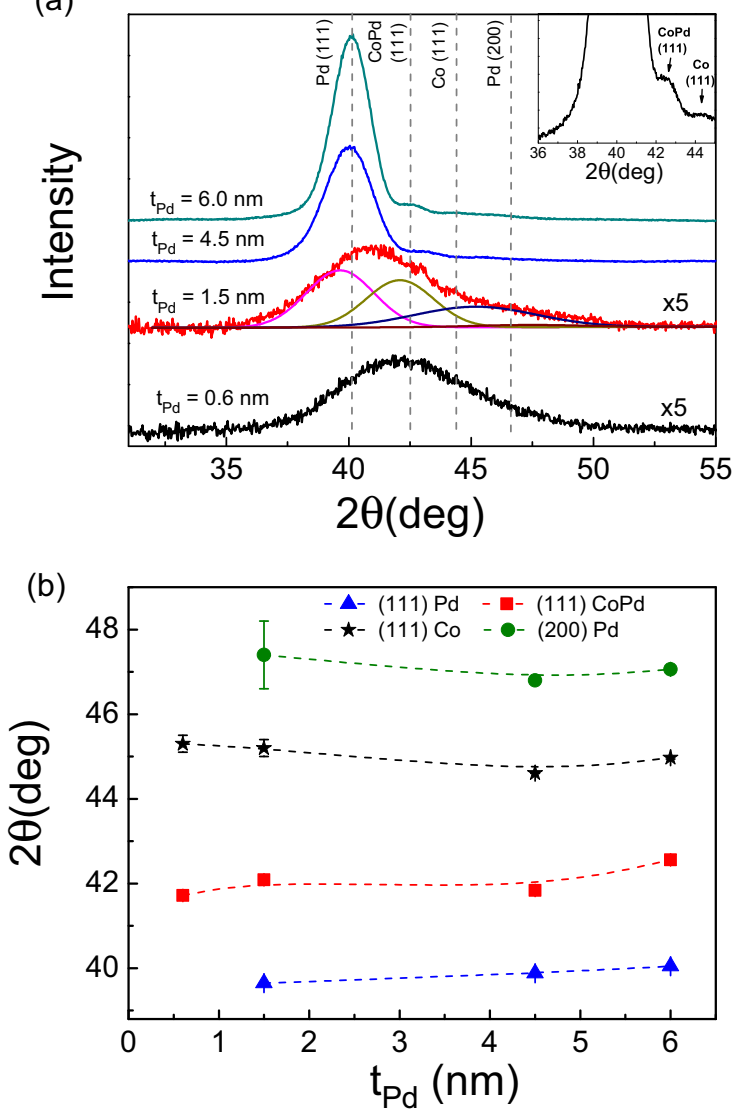

FIG. 3. (a) X-ray diffraction patterns of the Co-Pd granular films on glass $\left(t_{\mathrm{Pd}}=0.6,4.5\right.$, and $\left.6.0 \mathrm{~nm}\right)$ and on crystal silicon $\left(t_{\mathrm{Pd}}=1.5 \mathrm{~nm}\right)$. Reference crystalline diffractions positions of Co-Pd alloy [12,34], fcc Pd [33], and Co [37] are marked by dashed vertical lines. The corresponding fit for sample $t_{\mathrm{Pd}}=1.5 \mathrm{~nm}$ is indicated (see Supplemental Material for additional information). Inset: zoom of the XRD patterns for the samples with $t_{\mathrm{Pd}}=6.0 \mathrm{~nm}$. (b) Bragg positions as a function of the deposited Pd thickness.

$52^{\circ}$. No reference peak external to the multilayer was used to fix the zero shift; instead, the largest peak in the $t_{\mathrm{Pd}}=6.0 \mathrm{~nm}$ sample, which can be assigned unambiguously to (111) fcc Pd, was used as an internal reference. This is important to compare results from different film samples, whose diffraction zero shifts can be different. The positions of the diffraction peaks in the XRD profile are shown in Fig. 3(b). The refined values for the $2 \theta$ positions and the full width at half maximum (FWHM) are listed in Table A of the Supplemental Material [30]. Taking the (111) reflection of $\mathrm{Pd}$ in $t_{\mathrm{Pd}}=6.0 \mathrm{~nm}$ as reference, the refined $2 \theta$ values for samples with $t_{\mathrm{Pd}}=4.5 \mathrm{~nm}$ and $1.5 \mathrm{~nm}$ were shifted $+0.2^{\circ}$ and $+0.4^{\circ}$, respectively. The FWHM values, after corrections of the instrumental broadening, are compatible with NPs of a few $\mathrm{nm}$ diameter according to Scherrer formula [32].

We ascribed the diffraction peaks to Co-Pd phases as follows:

(a) The peak at $2 \theta \approx 40^{\circ}$ was clearly identified as the (111) reflection of fcc Pd (Ref. [33]), but highly textured with this plane perpendicular to the sample's growth direction, since the (200) reflection expected at $\approx 46.7^{\circ}$ is not observed. However, the (111) reflection of unalloyed Pd could not be resolved in the $t_{\mathrm{Pd}}=0.6 \mathrm{~nm}$ sample, in agreement with the Pd EDS map shown in the previous section.

(b) The peak at $\approx 42^{\circ}$ was assigned to a $\mathrm{Co}_{x} \mathrm{Pd}_{1-x}$ alloy and indexed as (111) in a cubic lattice. The cubic cell parameter derived from the diffraction angle can be related to the $\mathrm{Pd}$ concentration in the alloy [34], yielding $x$ values in the range $0.5-0.7$. Since the expected position of the (111) reflection of the tetragonal phase of CoPd is $43.9^{\circ}$ in thin films [35], we can discard $\mathrm{CoPd}$ with a long range order of the $\mathrm{L} 1_{0}$ tetragonal structure type.

(c) The assignment of the peak in the $44^{\circ}-46^{\circ}$ range was not straightforward. Discarding a tetragonal CoPd phase with long range crystalline order, only the (111) reflection from fcc $\mathrm{Co}$ is expected to contribute at $44.2^{\circ}$, if present [35]. The assignment of the peak fitted in the $t_{\mathrm{Pd}}=0.6 \mathrm{~nm}$ and $t_{\mathrm{Pd}}=1.5 \mathrm{~nm}$ patterns at $\approx 45.2^{\circ}$ to fcc Co would imply a contracted structure by $2 \%$ with respect to Ref. [36], with cell parameter $a=3.47 \AA$ and $d_{\mathrm{Co}-\mathrm{Co}}=2.45 \AA$. However, in these unstructured patterns with poor statistics, we cannot rule out contributions from higher angles, as the (200) reflection of not completely textured $\mathrm{Pd}$ or Co-Pd phases like $\mathrm{CoPd}$ and $\mathrm{CoPd}_{3}$, all in the $46^{\circ}-48^{\circ}$ range. Although pure Co is not clearly resolved, the patterns of the low Pd content samples suggest that some Co remains unalloyed.

\section{XANES and EXAFS measurements}

\section{Co K edge}

The X-ray absorption spectra (XAS) at the Co $K$ edge for Co-Pd granular films with $t_{\mathrm{Co}}=0.7 \mathrm{~nm}$ and $t_{\mathrm{Pd}}=0.6,1.5,4.5$, and $6.0 \mathrm{~nm}$ are shown in Fig. 4(a), along with those of bare fcc Co NPs $\left(t_{\mathrm{Co}}=0.7 \mathrm{~nm}\right)$ and bulk $\mathrm{Co}_{50} \mathrm{Pd}_{50}$ alloy, plotted for comparison. The XAS signal, $\mu(E)$, was analyzed using different tools of the IFFEFIT XAS package [38].

The intensity and shape of the shoulder [denoted as I in Fig. 4(a)] and the white line (denoted as II) of the normalized $\mathrm{x}$-ray absorption near edge structure (XANES) reflect a population rearrangement between the electronic levels of $\mathrm{Co}$ and Pd upon alloying $[39,40]$. The structure within the edge is more obvious in the first derivative of the XANES spectrum plotted in Fig. 4(b). In order to analyze these features more clearly, we calculated the difference between the areas of the peaks I and II with respect to that of the Co bare NPs as

$$
\Delta A_{\mathrm{I}(\mathrm{II})}=\int_{E_{i, \mathrm{I}(\mathrm{II})}}^{E_{f, \mathrm{III})}}\left(\mu_{\text {sample }}-\mu_{\mathrm{Co}-\mathrm{bareNPS}}\right) d E,
$$

which is plotted as a function of the thickness of deposited $\mathrm{Pd}$ content in the inset of Fig. 4(a). The integration limits were set at the energies on both sides of the shoulder I and the peak II features in the first derivative [Fig. 4(b)]. Positive $\Delta A_{\mathrm{I}(\mathrm{II})}$ indicates that the absorption is stronger in the Co-Pd samples than in the Co NPs, while negative values appear for the opposite situation.

Since the pre-edge quadrupolar transitions are expected to be too weak to be detected, $3 d$ band contributions to the Co $K$ edge XANES spectra ( $1 s \rightarrow 4 p$ transitions) can be observed in the pre-edge only through $3 d-4 p$ hybridization, which is reflected as a shoulder (peak I) [41]. Although alloying with 


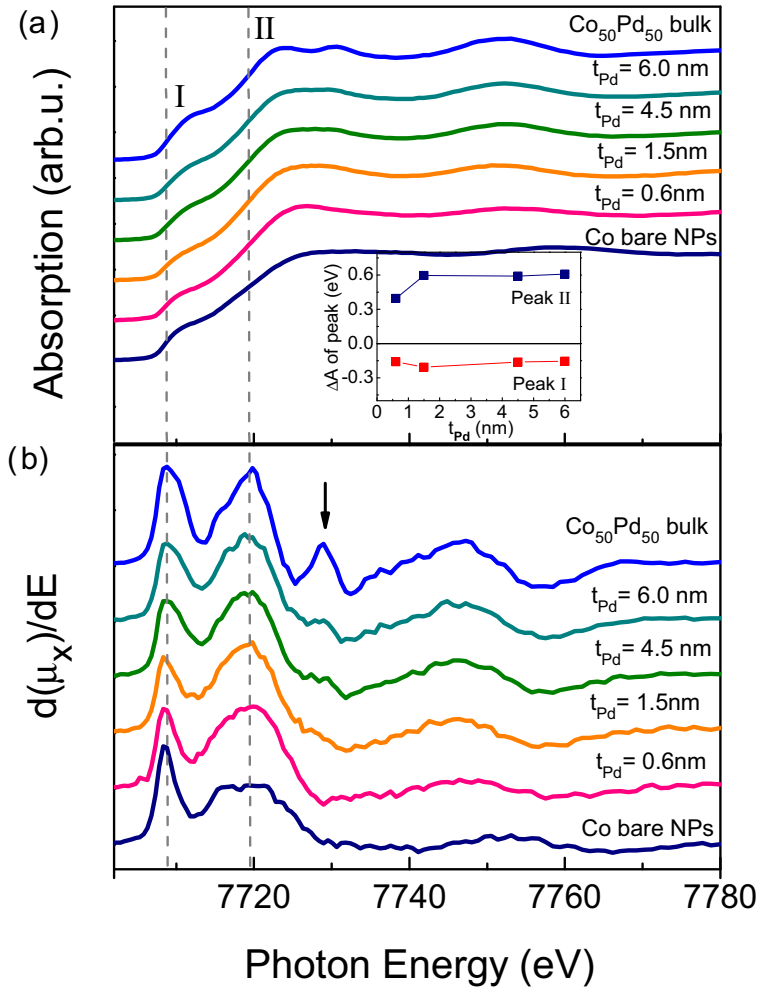

FIG. 4. (a) XANES spectra at the Co $K$ edge for the CoPd NPs and those of the $\mathrm{Co}$ foil and $\mathrm{Co}_{50} \mathrm{Pd}_{50}$ bulk alloy, plotted for reference. Inset: area difference of peaks I and II with respect to Co bare NPs, as a function of the Pd concentration. (b) First derivative plots of Co $K$ edge XANES spectra shown in (a). The meaning of the vertical arrow is explained in the main text.

Pd actually increases the number of $d$ holes in the alloy's band structure, hybridization induced by the $\mathrm{Pd} 4 d$ states would delocalize these $d$ empty states and, as a consequence, reduce the density of empty $d$ states projected onto the Co site [41]. Indeed, we may see that the intensity of peak I in the CoPd multilayers is reduced with respect to that of the bare Co NPs (negative $\Delta A_{\mathrm{I}}$ ), proving the effect of $\mathrm{Pd}$ on the Co $3 d-\mathrm{Pd} 4 d$ hybridization.

The Co $K$ edge white line, peak II, also provides information on the modifications introduced by alloying. The white

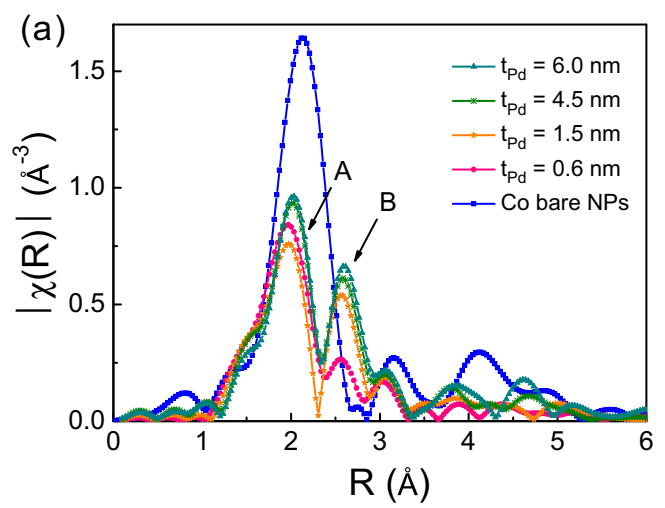

line intensity depends on both the number of available $4 p$ states for the $1 s \rightarrow 4 p$ transitions and the structure of the system. In Fig. 4(a) we observe a higher intensity of peak II in all CoPd multilayers than that in the Co bare NPs. Moreover, the shape is different, displaying modifications in the local structure of the Co-Pd alloy in the NPs of the granular system with respect to the fcc Co of the Co bare NPs. While in peak I the area difference $\Delta A_{\mathrm{I}}$ is negative and is nearly constant along the multilayer series, $\Delta A_{\mathrm{II}}$ is positive, increases $20 \%$ from $t_{\mathrm{Pd}}=0.6 \mathrm{~nm}$ to $1.5 \mathrm{~nm}$, and attains a constant value for increasing $\mathrm{Pd}$ thickness. Therefore, the number of Co $4 p$ empty states increases with alloying and then reaches a constant value.

The XANES spectrum of the $\mathrm{Co}_{50} \mathrm{Pd}_{50}$ bulk alloy (Fig. 4) is similar to those of the Co-Pd NPs with higher Pd content, but differs from those of the lowest Pd content or Co bare NPs. In effect, in $\mathrm{Co}_{50} \mathrm{Pd}_{50}$ the white line is split in two in the 7720-7735 eV range, and has a large amplitude in the first EXAFS oscillation. The right-side part of the splitting is neatly reflected in the first derivative as a peak at $7730 \mathrm{eV}$ [marked with an arrow in Fig. 4(b)], while the oscillation is evident at $\approx 7750 \mathrm{eV}$. The white line splitting, though in a less definite way, is present in all the samples, except for $t_{\mathrm{Pd}}=0.6 \mathrm{~nm}$. However, this sample clearly shows EXAFS features intrinsic to Co-Pd systems in the 7740-7750 eV range. Therefore, Co-Pd alloying is progressive as $\mathrm{Pd}$ is added, and it is complete at a Pd deposition thickness above $1.5 \mathrm{~nm}$.

The local structure of the CoPd granular films was studied from the EXAFS signal, $\chi(k)$. Figure 5 shows the Fourier transform (FT) of the Co $K$ edge EXAFS signal. An intense peak at $R \sim 2 \AA$ (peak A in Fig. 5) is present in all samples, including the reference bulk alloys, while a weaker one at $R \sim 2.6 \AA$ (peak B in Fig. 5) is absent in the Co film and Co bare NPs. Therefore, this second peak is produced by the presence of the Pd nearest neighbors. Moreover, the positions and relative heights of both peaks are similar to those of $\mathrm{Co}_{50} \mathrm{Pd}_{50}$, except for the multilayer with $t_{\mathrm{Pd}}=0.6 \mathrm{~nm}$, which shows similarities to alloys richer in Co.

The data reduction and fits were performed in all reference and CoPd multilayer samples, using the ARTEMIS code [38], and crystallographic models based on cell parameter data from the ICSD database [42]. FT into $R$ space was performed in a $k$ range from 2.4 to $10.6 \AA^{-1}$, using $k^{2}$ weight and a Hanning

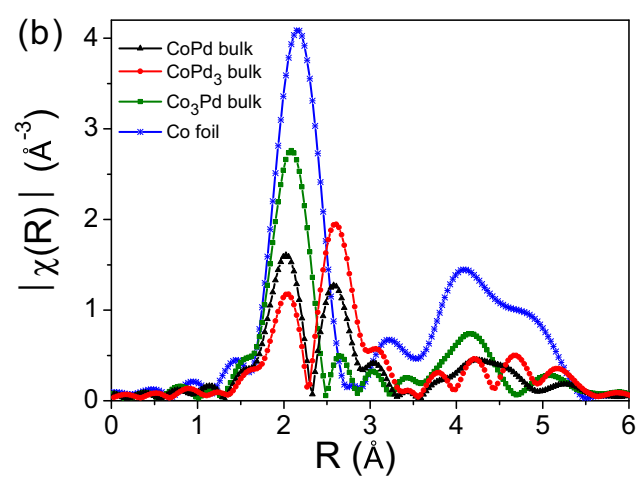

FIG. 5. (a) FT of the Co $K$ edge EXAFS signal of the Co-Pd samples and Co bare NPs with $t_{\mathrm{Co}}=0.7$ nm included for comparison. (b) Comparison of the FT of the EXAFS signal of $\mathrm{Co}_{3} \mathrm{Pd}$, $\mathrm{CoPd}$, and $\mathrm{CoPd}_{3}$ bulk reference samples and Co foil. 


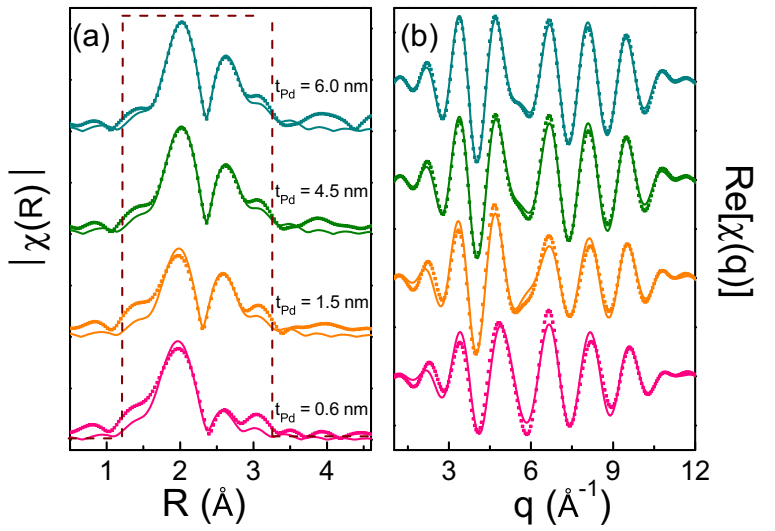

FIG. 6. Contribution of the first coordination shell to the EXAFS signal at the Co $K$ edge for the CoPd NPs with the best fits plotted in (a) $R$ space and (b) as filtered EXFS signal. Symbols represent the experimental data and solid lines, the best fits. Curves have been vertically shifted for clarity.

window. Fits were performed in a $R$ range from 1.2 to $3.2 \AA$. Both the tetragonal ordered structure $\mathrm{L} 1_{0}$ and the chemically disordered A1 phase were tried; for the sample with $t_{\mathrm{Pd}}=$ $0.6 \mathrm{~nm}$ also the $\mathrm{L}_{2}$ structure of $\mathrm{Co}_{3} \mathrm{Pd}$ was tried. The fitting parameters for each sample were the average coordination number $(N)$, the interatomic distance $(R)$, and the DebyeWaller factor $\left(\sigma^{2}\right)$, for each path. The amplitude reduction factor $S_{0}^{2}$ was fixed to 0.75 , which was the value obtained for the fit of the CoPd bulk reference sample. The threshold energy $\Delta E_{0}$ was set to $2.5 \mathrm{eV}$ for samples with $t_{\mathrm{Pd}}=0.6$ and $1.5 \mathrm{~nm}$, and $4 \mathrm{eV}$ for samples with $t_{\mathrm{Pd}}=4.5$ and $6.0 \mathrm{~nm}$.

While fit attempts with the $\mathrm{A} 1$ or $\mathrm{L}_{2}$ phase were not successful, fits using the tetragonalized cell with lattice parameter ratio $c / a \sim 1$ yielded consistent results, but they were worse for the samples with lower Pd thickness. The fits for the Co-Pd NPs are shown in Fig. 6, while the refined parameters are listed in Table I. Both Co-Co and Co-Pd distances are very similar for all the samples (although slightly smaller for $t_{\mathrm{Pd}}=0.6$ $\mathrm{nm}$ ), which reflects that the alloying present in each sample has nearly the same composition, in agreement with the Co $K$ edge XANES and XRD results. Besides, these distances are close to the theoretically estimated values for $\mathrm{Co}_{50} \mathrm{Pd}_{50}$ clusters at the Co $K$ edge [43]. The Debye-Waller factors are rather high for both Co-Co and Co-Pd bonds in all samples, which can be attributed to dispersion of atom-atom distances because of the finite particle size. The element specific ratio $(c / a)_{\mathrm{Co}}=$ $\sqrt{2\left(R_{\mathrm{Co}-\mathrm{Pd}} / R_{\mathrm{Co}-\mathrm{Co}}\right)^{2}-1}$ at the Co atoms in $\mathrm{L} 1_{0}$ unit cell [39] is $\approx 1.08$. This is larger than that measured in bulk $\mathrm{L}_{0} \mathrm{CoPd}$, $c / a \sim 0.97$ [44], but it is similar to that found in the CoPt NP multilayers [21]. This has been explained as relaxation of the atom-atom distances because of finite size in the $\mathrm{L} 1_{0}$ structure, and DFT calculations for finite size Co-Pt relaxed clusters effectively predict a ratio $c / a($ EXAFS $)>1$ [39]. Therefore, the present CoPd NPs in the fabricated multilayers could also show this structural relaxation.

The fitted Co-Pd coordination number for the $t_{\mathrm{Pd}}=0.6 \mathrm{~nm}$ sample is very small. Although the shape of the FT suggested a possible $\mathrm{Co}_{3} \mathrm{Pd}$ phase, this was not supported by the fits. Alternatively, the high Co-Co path contribution to $|\chi(R)|$ with respect to that of Co-Pd paths may reflect a core-shell structure of the NPS with an fcc Co core and a $\mathrm{L} 1_{0}$ CoPd shell. Then, the analysis with a single $\mathrm{L}_{0}$ phase would produce phaseaveraged coordination numbers and interatomic distances; in fact, the slightly short fitted Co-Co distance for this sample is consistent with a Co core of shorter Co-Co distances than those in the $\mathrm{L} 1_{0}$ phase, supporting the indications of such a core in the XRD analysis.

\section{Pd K edge}

The normalized XANES spectra at the Pd $K$ edge for the CoPd NPs are shown in Fig. 7, together with that of a Pd foil for comparison. The hybridization-mediated $1 s \rightarrow 4 d, d p$ absorption transition in the pre-edge region is unresolved in the $K$-edge spectra of heavier $4 d$ elements as Pd, and it is only observed as a shoulder that can be marked as the first inflection point in the pre-edge (Fig. 7) $[45,46]$. The two resonance peaks after the pre-edge are due to transitions $1 s \rightarrow 5 p$ and $1 s \rightarrow 4 f$ also through hybridization, respectively. The spectrum of the sample with the largest amount of deposited Pd $\left(t_{\mathrm{Pd}}=6.0 \mathrm{~nm}\right)$ is very similar to that of the Pd foil, and its slightly smaller amplitude reduction can be ascribed to size effects as in Pd nanoparticles [47]. As $t_{\mathrm{Pd}}$ decreases, the amplitude of the XANES and EXAFS peaks decreases, which can reflect increasing size effects as well as reveal electronic effects intrinsic to the Co-Pd alloying. However, those two effects cannot be distinguished in the XANES spectra.

The EXAFS signal of the Pd $K$ edge was analysed in a similar way to that of the Co edge. Experimental and fitting details together with figures are included in the Supplemental Material [48], and the fitted parameters are included here in Table II. The refined Pd-Co distances are in good agreement with those obtained in the Co $K$ edge EXAFS analysis, including the slightly smaller distance in the $t_{\mathrm{Pd}}=0.6 \mathrm{~nm}$ sample (see Table I), which confirms the presence of the

TABLE I. Structural parameters obtained from the Co $K$-edge EXAFS fits for the CoPd NPs shown in Fig. 5. Coordination numbers $N$, interatomic distance $R$, and Debye-Waller factor $\sigma^{2}$, and $c / a$ ratio for each path used in the fitting model.

\begin{tabular}{lccccccc}
\hline \hline & \multicolumn{9}{c}{$\mathrm{Co}-\mathrm{Co}$} & \multicolumn{2}{c}{$\mathrm{Co}-\mathrm{Pd}$} \\
$n_{\mathrm{Pd}}(\mathrm{nm})$ & $N_{\mathrm{Co}-\mathrm{Co}}$ & $R_{\mathrm{Co}-\mathrm{Co}}(\AA)$ & $\sigma_{\mathrm{Co}-\mathrm{Co}}^{2}\left(\AA^{2}\right)$ & $N_{\mathrm{Co}-\mathrm{Pd}}$ & $R_{\mathrm{Co}-\mathrm{Pd}}\left(\AA^{2}\right)$ & $\sigma_{\mathrm{Co}-\mathrm{Pd}}^{2}\left(\AA^{2}\right)$ \\
\hline 0.6 & $4.2(8)$ & $2.46(3)$ & $0.013(2)$ & $2.6(9)$ & $2.57(3)$ & $0.010(1)$ & $1.087(5)$ \\
1.5 & $4.1(6)$ & $2.50(3)$ & $0.011(1)$ & $3.9(8)$ & $2.59(1)$ & $0.015(2)$ & $1.071(6)$ \\
4.5 & $3.9(3)$ & $2.49(1)$ & $0.012(2)$ & $4.9(7)$ & $2.61(1)$ & $0.013(2)$ & $1.094(2)$ \\
6.0 & $3.5(8)$ & $2.49(2)$ & $0.010(1)$ & $5.9(8)$ & $2.61(1)$ & $0.014(1)$ & $1.094(2)$ \\
\hline \hline
\end{tabular}




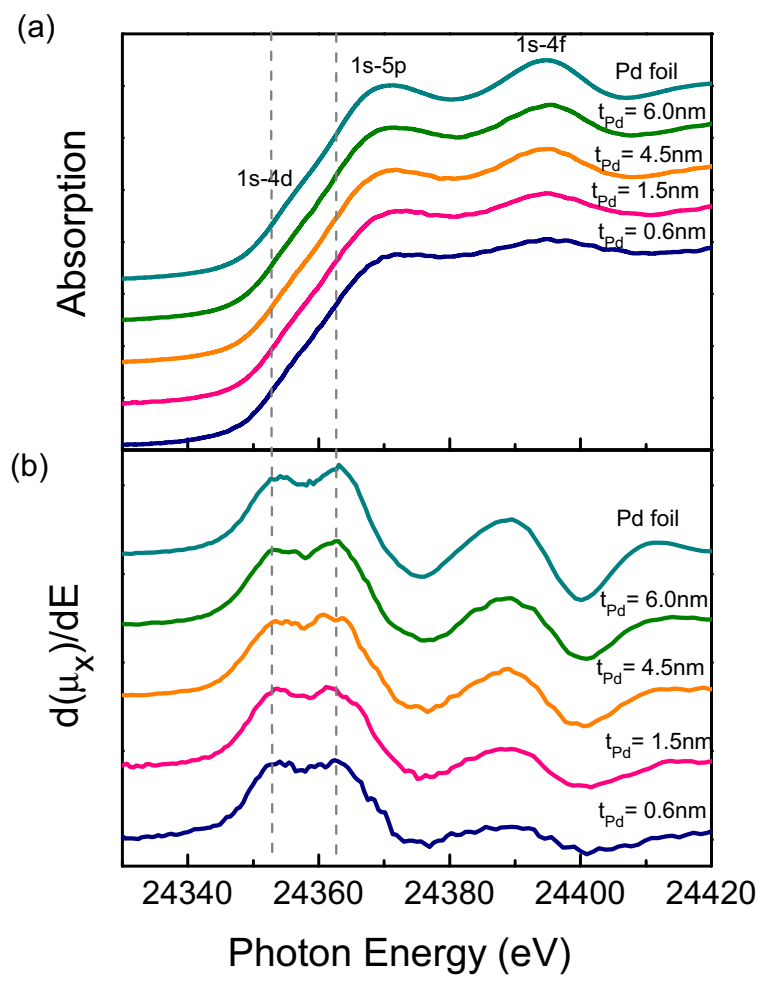

FIG. 7. (a) XANES spectra at the Pd $K$ edge for the Co-Pd granular multilayer samples and $\mathrm{Pd}$ foil sample, plotted for reference. The spectra are shifted in the intensity scale to ease comparison. Inset: the spectra after the absorption edge jump in the $24375-24450 \mathrm{eV}$ range without shifts in the intensity scale. (b) First derivative plots of Pd $K$ edge XANES spectra presented in (a).

Co-Pd alloy in the samples. In fact, the ratio $(c / a)_{\mathrm{Pd}}=$ $\sqrt{2\left(R_{\mathrm{Co}-\mathrm{Pd}} / R_{\mathrm{Pd}-\mathrm{Pd}}\right)^{2}-1}$ at the $\mathrm{Pd}$ atoms is now about 0.92 , in agreement with the ratio $(c / a)_{\text {Co }}>1$ at the Co atoms [39]. Moreover, these values are comparable with those theoretically estimated for $\mathrm{Co}_{50} \mathrm{Pd}_{50}$ clusters at $\mathrm{Pd} K$ edge $[43,49]$. Note that the Pd-Pd coordination number systematically increases, and the Debye-Waller factor for the Pd-Pd distance decreases, with the amount of $\mathrm{Pd}$ in the system, which reflects the increasing amount of metallic Pd. Unfortunately, this limits the reliability of the fitted coordination numbers, which were derived from a two phase system using a single phase fit, except probably for the sample with the lowest Pd thickness; indeed, the value $N_{\mathrm{Pd}-\mathrm{Co}}=5.0$ for $t_{\mathrm{Pd}}=0.6 \mathrm{~nm}$ is consistent with the values $N_{\mathrm{Co}-\mathrm{Pd}}$ obtained from the $\mathrm{K}$ edge Co EXAFS (Table I).

\section{MAGNETIC PROPERTIES}

\section{A. SQUID magnetometry}

The four samples were zero field cooled (ZFC) or field cooled (FC) down to $5 \mathrm{~K}$ under a $0.1 \mathrm{kOe}$ bias magnetic field applied parallel or perpendicular to the samples' plane. Then, the respective thermal magnetization curves were measured with different applied field values, $H_{\mathrm{dc}}$. Figures $8(\mathrm{a})$ and (b) show $M(T)$ of the samples with $t_{\mathrm{Pd}}=1.5$ and $6.0 \mathrm{~nm}$, respectively, for both field directions. When the applied field is perpendicular to the sample's plane, three temperature regions from the ZFC curve can be identified as the temperature increases: an initial increase in $M_{\perp}(T)$, a change in its slope, and its coalescence with the FC $M_{\perp}(T)$.

There is a concomitant behavior in the temperature dependence of the hysteresis loops. These were measured in both orthogonal configurations of the magnetic field at temperatures in the different magnetic regions. Figure 9 shows the hysteresis curves for the samples with $t_{\mathrm{Co}}=0.7 \mathrm{~nm}$ and $t_{\mathrm{Pd}}=1.5 \mathrm{~nm}$, as an example. At low temperature, PMA was identified for all the samples; the square shape of the $M_{\perp}(H)$ loop displays hard ferromagnetic behavior along the easy axis of magnetization, while the shape, the coercivity, and the remanence of the $M_{\|}(H)$ loop correspond to a magnetic hard axis. In the temperature region above the change in slope of the ZFC magnetization, coercivity is almost zero, although the easy axis direction of the magnetization remains perpendicular to the substrate plane, i.e., the multilayer behaves as a soft ferromagnet with PMA [Fig. 9(b)]. Finally at higher temperatures, $M(H)$ increases monotonously and displays no anisotropy, as expected for a superparamagnetic system of noninteracting nanoparticles [Fig. 9(c)]. Figure 10(a) shows the temperature dependence of coercivity for the perpendicular applied field direction for all samples. Note that $H_{\mathrm{C} \perp}$ reaches values as high as $8.9 \mathrm{kOe}$ at $5 \mathrm{~K}$, and $0.3 \mathrm{kOe}$ at $250 \mathrm{~K}$ for the sample with the highest amount of $\mathrm{Pd}$.

The hysteresis loops along with the FC and ZFC curves can be used to define two temperatures associated to changes in the magnetic behavior. The temperature $T_{1}$ was defined as that at which coercivity vanishes, while $T_{f}$ was determined from the coalescence of the FC and ZFC $M_{\perp}(T)$ curves. ZFC curves measured under different perpendicular $H_{\mathrm{dc}}$ fields show that increasing the field leads to a decrease in $T_{1}$, tending to zero at an extrapolated field value of $\approx 5 \mathrm{kOe}$ in $M_{\perp}(T)$ [Fig. 8(c)]. Similarly, when the applied magnetic field is parallel to the substrate plane [Fig. 8(d)], $T_{f}$ is also observed to shift to lower temperature with increasing applied field until the maximum in $M_{\|}(T)$ becomes constant value at low temperature, which

TABLE II. Structural parameters obtained from the Pd $K$ edge EXAFS fits for the Co-Pd NPs (figures are included in the Supplemental Material [48]). Coordination number $N$, interatomic distance $R$, and Debye-Waller factor $\sigma^{2}$ for both paths used in the fitting model.

\begin{tabular}{|c|c|c|c|c|c|c|}
\hline \multirow[b]{2}{*}{$t_{\mathrm{Pd}}(\mathrm{nm})$} & \multicolumn{3}{|c|}{ Pd-Co } & \multicolumn{3}{|c|}{$\mathrm{Pd}-\mathrm{Pd}$} \\
\hline & $N_{\mathrm{Pd}-\mathrm{Co}}$ & $R_{\mathrm{Pd}-\mathrm{Co}}(\AA)$ & $\sigma_{\mathrm{Pd}-\mathrm{Co}}^{2}\left(\AA^{2}\right)$ & $N_{\mathrm{Pd}-\mathrm{Pd}}$ & $R_{\mathrm{Pd}-\mathrm{Pd}}(\AA)$ & $\sigma_{\mathrm{Pd}-\mathrm{Pd}}^{2}\left(\AA^{2}\right)$ \\
\hline 0.6 & $5.0(2)$ & $2.56(2)$ & $0.015(1)$ & $6.3(9)$ & $2.69(1)$ & $0.012(2)$ \\
\hline 1.5 & $4.3(2)$ & 2.61(1) & $0.015(2)$ & $7.9(9)$ & 2.71(1) & $0.008(1)$ \\
\hline 4.5 & $3.2(5)$ & $2.64(3)$ & $0.018(3)$ & $9.5(5)$ & $2.72(1)$ & $0.008(1)$ \\
\hline 6.0 & $2.3(9)$ & $2.64(3)$ & $0.022(2)$ & $10.5(7)$ & $2.73(1)$ & $0.007(1)$ \\
\hline
\end{tabular}



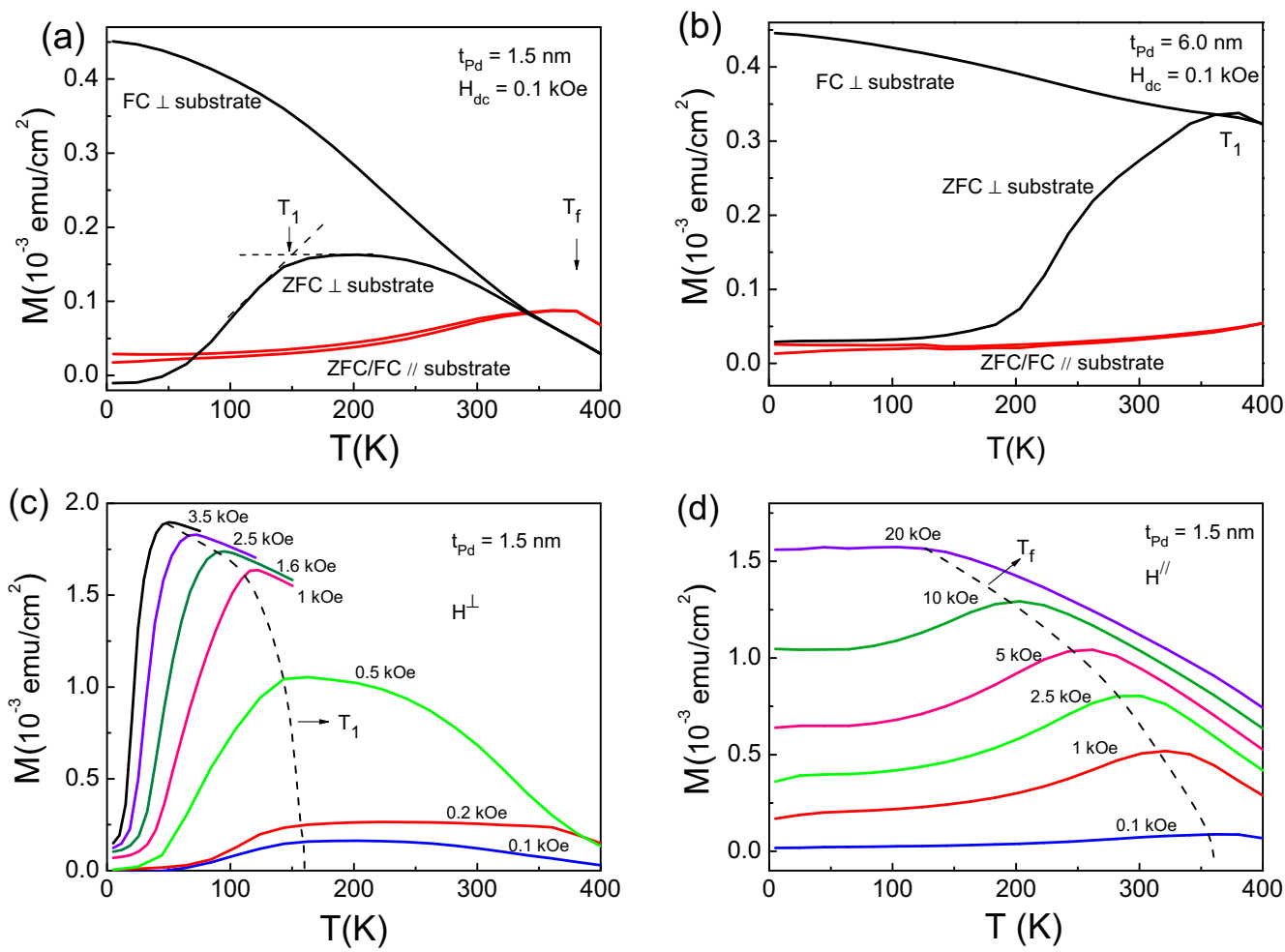

FIG. 8. $M(T)$ curves measured under an applied field $H_{\mathrm{dc}}=0.1 \mathrm{kOe}$ parallel $(\|)$ and perpendicular $(\perp)$ to the substrate plane for the samples with (a) $t_{\mathrm{Pd}}=1.5 \mathrm{~nm}$, and (b) $t_{\mathrm{Pd}}=6.0 \mathrm{~nm}$. $M(T)$ curves measured at different applied fields values, $H_{\mathrm{dc}}$ indicated beside each curve, in the (c) $\perp$ and (d) $\|$ direction for the sample with $t_{\mathrm{Pd}}=1.5 \mathrm{~nm}$. Dashed lines (--) show the dependence of $T_{1}$ and $T_{f}$ with $H_{\mathrm{dc}}$.
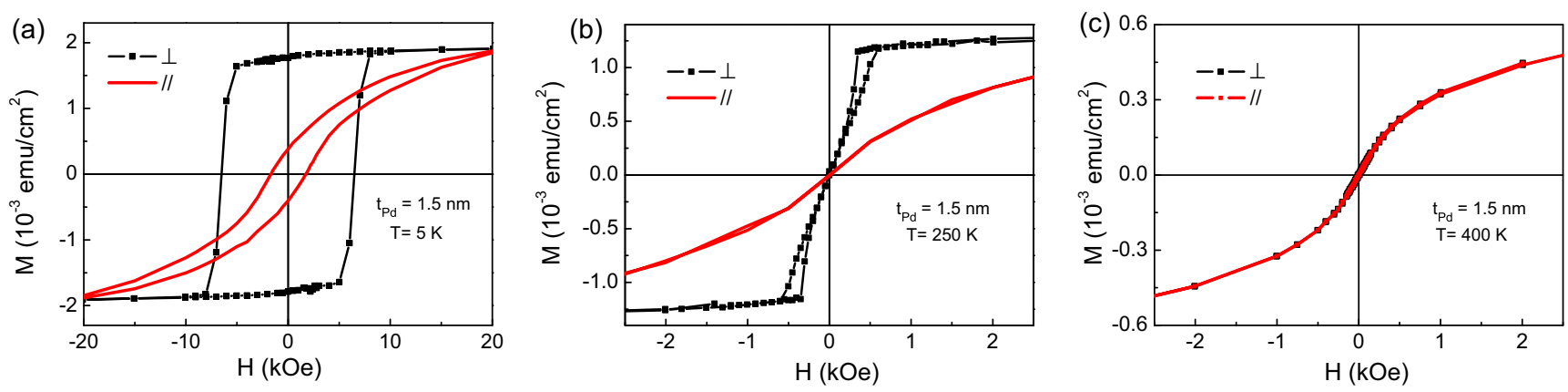

FIG. 9. Magnetization curves measured for the sample with $t_{\mathrm{Pd}}=1.5 \mathrm{~nm}$ under both parallel and perpendicular applied field at (a) $5 \mathrm{~K}$, (b) $250 \mathrm{~K}$, and (c) $350 \mathrm{~K}$.
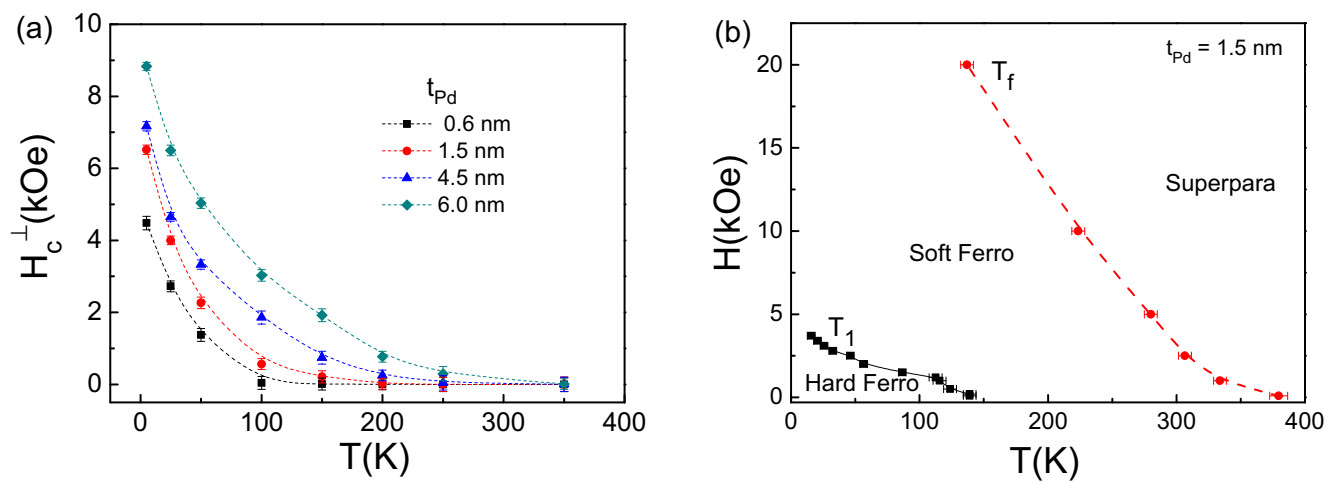

FIG. 10. (a) Temperature dependence of the coercive field when the magnetic field is perpendicular to the substrate plane for all the samples. (b) Magnetic phase diagram of the CoPd NPs with $t_{\mathrm{Pd}}=1.5 \mathrm{~nm}$. 
TABLE III. Summary of parameters deduced from the magnetic measurements at $5 \mathrm{~K}$. Coercive fields $H_{\mathrm{C} \perp}$ and $H_{\mathrm{C} \|}$, saturation magnetization $M_{\mathrm{S}}$, and effective anisotropy constants $K_{\text {eff }} t$ for the full sample (25 layers) and $K_{\text {eff }}$ (referred to $0.7 \mathrm{~nm}$ of Co). $K_{\text {eff }}^{*}$ was calculated using layer thickness measured from TEM images. Transition temperatures $T_{1}$ and $T_{f}$ obtained from ZFC curves at $H_{\mathrm{dc}}=0.1 \mathrm{kOe}$.

\begin{tabular}{lcccccccc}
\hline \hline $\begin{array}{l}t_{\mathrm{Pd}} \\
(\mathrm{nm})\end{array}$ & $\begin{array}{c}T_{1} \\
(\mathrm{~K})\end{array}$ & $\begin{array}{c}T_{f} \\
(\mathrm{~K})\end{array}$ & $\begin{array}{c}H_{\mathrm{C} \perp} \\
(\mathrm{kOe})\end{array}$ & $\begin{array}{c}H_{\mathrm{C} \|} \\
(\mathrm{kOe})\end{array}$ & $\begin{array}{c}M_{\mathrm{S}} \\
\left(10^{-3} \mathrm{emu} / \mathrm{cm}^{2}\right)\end{array}$ & $\begin{array}{c}K_{\text {eff }} t \\
\left(\mathrm{erg} / \mathrm{cm}^{2}\right)\end{array}$ & $\begin{array}{c}K_{\text {eff }} \\
\left(10^{7} \mathrm{erg} / \mathrm{cm}^{3}\right)\end{array}$ & $\begin{array}{c}K_{\text {eff }}^{*} \\
\left(10^{7} \mathrm{erg}^{2} \mathrm{~cm}^{3}\right)\end{array}$ \\
\hline 0.6 & $120(10)$ & $325(5)$ & $4.5(1)$ & $2.3(2)$ & $1.9(1)$ & $11(1)$ & $0.60(3)$ & $0.14(3)$ \\
1.5 & $200(10)$ & $350(5)$ & $6.5(2)$ & $1.7(1)$ & $1.9(1)$ & $21(2)$ & $7.2(3)$ & $0.24(3)$ \\
4.5 & $300(10)$ & $325(5)$ & $7.2(1)$ & $0.9(3)$ & $3.3(2)$ & $75(8)$ & $4.3(5)$ & $0.48(5)$ \\
6.0 & $350(10)$ & $375(5)$ & $8.9(1)$ & $0.9(2)$ & $3.4(2)$ & $53(5)$ & $3.0(3)$ & $0.28(3)$ \\
\hline \hline
\end{tabular}

means that all the particles in the film are fully polarized along the applied field.

The dependence of the temperatures $T_{1}$ and $T_{f}$ with the applied field allows us to draw a temperature-magnetic field phase diagram [Fig. 10(b)], which will be discussed later. A similar magnetic phase diagram has been obtained for the $\mathrm{CoPt}$ NPs [21]. Nevertheless, the highest $T_{1}$ reported in the case of capping with Pt is $\sim 140 \mathrm{~K}$, while for CoPd NPs the highest $T_{1}$ is $\sim 280 \mathrm{~K}$ at $H_{\mathrm{dc}}=0.1 \mathrm{kOe}$ (Table III).

The coercivity for magnetic fields applied parallel or perpendicular to the sample's plane shows an increase in $H_{\mathrm{C} \perp}$ with the Pd thickness, while $H_{\mathrm{C} \|}$ decreases. This reflects an increase in the magnetic hardness, which can also be quantified as an effective magnetic anisotropy constant, $K_{\text {eff }}$ in the hard magnetic phase. The $K_{\text {eff }}$ can be calculated from the area between the anhysteretic magnetization in the first quadrant measured perpendicular and parallel to the sample surface $[13,21,50]$. However, since what is directly measured is the magnetic moment of the multilayer sample, the magnetic moment per sample's unit area yields the quantity $K_{\text {eff }} t$ for the present samples, where $t$ is the effective magnetic thickness in the Co-Pd multilayers (see Sec. III A). We found values of $K_{\text {eff }} t$ in the range 11 to $75 \mathrm{erg} / \mathrm{cm}^{2}$ (Table III). It should be noticed that a derivation of the actual value of $K_{\text {eff }}$ relies on the knowledge of the effective thickness $t$, which may be ambiguous as along as the actual extent of the multilayer contributing magnetically is unknown. Here we used for $t$ the nominal deposited thickness of Co $(0.7 \mathrm{~nm})$, although some polarized Pd (see Sec. IV B 2) can contribute to $t$ and some Co atoms are known not to be in the Co nanoparticles and are paramagnetic. For comparison with the previous work on the Pt multilayers [21,51], the anisotropy constant derived from the CoPd layer thickness $t$ measured from TEM images is also included in Table III, which lists the magnetic parameters $H_{c}$, $M_{s}, K_{\text {eff }} t$, and $K_{\text {eff }}$ at $5 \mathrm{~K}$ for all samples.

\section{B. X-ray magnetic circular dichroism}

\section{Co $K$ and $L_{2,3}$ edges}

The XMCD signal at the Co $K$ edge probes the $4 p$ empty states of the Co atoms in the sample. Since the signal is nonzero, it reflects the magnetic polarization of the Co $4 p$ band caused by the intra-atomic exchange interaction with the $3 d$ band. The spectrum recorded on the sample with $t_{\mathrm{Pd}}=1.5 \mathrm{~nm}$ is shown in Fig. 11, normalized to the absorption jump obtained for the Co $K$ edge XAS signal after background removal. Three features characterize the shape of the XMCD signal, namely two minima $\mathrm{A}$ and $\mathrm{B}$, and a maximum at higher energy $\mathrm{D}$.
In the absence of XMCD measurements on a CoPd alloy in bulk or thin film sample, we compared with the Co $K$ edge XMCD of CoPt and Co bare NPs spectra with the same $t_{\mathrm{Co}}=$ $0.7 \mathrm{~nm}$. Peaks A and B appear practically at the same photon energies in both alloyed samples, and they are directly related to the pre-peak I and the first minimum slope in the XANES spectrum (Fig. 4), respectively. In spite of the coincidence in energy, the relative intensities of A and B differ, since in the CoPd NPs case the amplitude of A is dominant. Moreover,the contribution from metallic Co seems to be very small in the current CoPd NP samples, since the intensity of the peak B is much smaller than that in Co bare NPs, but this peak reveals the presence of unalloyed Co. This suggests that alloying in the CoPd NPs is larger than in the CoPt case [21], and the Co core in the core-shell structure is smaller.

The XAS at the Co $L_{2,3}$ edges of the CoPd NPs with $t_{\mathrm{Pd}}=$ $1.5 \mathrm{~nm}$ was collected at an incident angle $\gamma=10^{\circ}$ with respect to the sample's surface normal [Fig. 12(a)]. The XMCD signal was normalized to the XAS spectrum in the high energy limit, after background subtraction [Fig. 12(b)]. From the magnetooptical sum rules [52,53], and neglecting the magnetic spin dipole moment in Co [53], the orbital to spin moment ratio

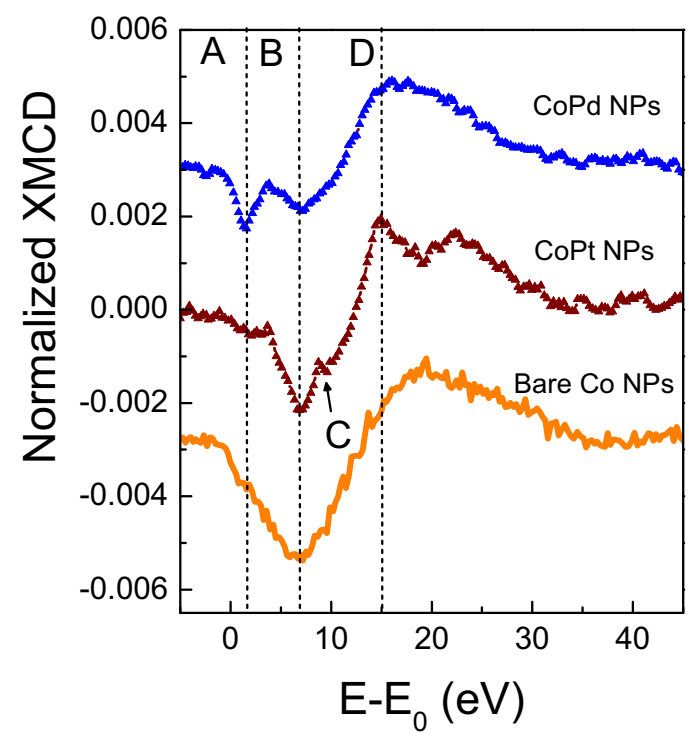

FIG. 11. Co $K$-edge XMCD signal measured in the CoPd NPs and comparison to that of CoPt NPs from Ref. [21] and of bare Co NPs with the same $t_{\text {Co }}$ from Ref. [20]. Curves were shifted vertically for the sake of clarity. 

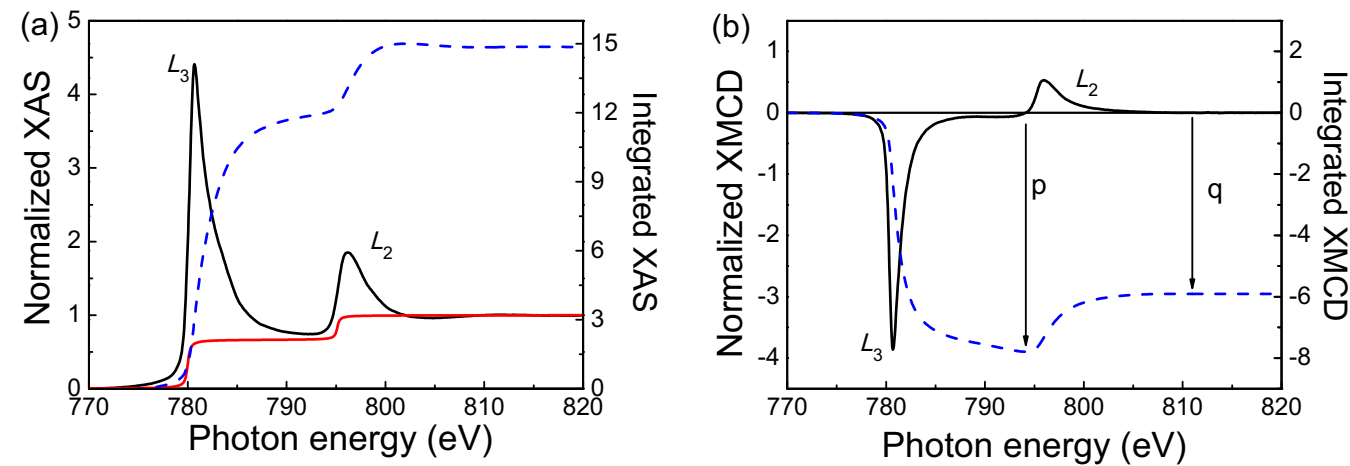

FIG. 12. Co $L_{2,3}$ edge spectra of the sample with $t_{\mathrm{Co}}=0.7 \mathrm{~nm}$ and $t_{\mathrm{Pd}}=1.5 \mathrm{~nm}$. (a) Normalized average XAS spectrum (solid line), double-step function used, and integrated white line (dashed line). (b) Normalized XMCD spectrum (solid line) and its integrated area (dashed line). Arrows mark the end energy limits of the integrals $p$ and $q$ used in the sum rules.

was directly derived as

$$
\frac{m_{\mathrm{L}}}{m_{\mathrm{S}}}=\frac{2 q}{(9 p-6 q)},
$$

where $q$ is the integrated area of the XMCD signal over the two edges and $p$ the integrated area over the $L_{3}$ edge only. This ratio is independent of the number of holes and of the normalization method used. We found $m_{\mathrm{L}} / m_{\mathrm{S}}=0.29(1)$.

The absolute values of the orbital and spin magnetic moments can be derived from the sum rules if the number of $3 d$ band holes in Co, $n_{h}$, is known. A value of $n_{h}=2.3$ was estimated with the FDMNES code for bulk $\mathrm{L}_{0}$ CoPd (see Sec. V below), although the multiple scattering theory and muffin-tin approximation used are not considered accurate to calculate the XMCD at the Co $L_{2,3}$ edges [54,55]. This value is slightly lower than 2.49 calculated for bulk Co [56] or 2.4 calculated for Co adatoms on $\mathrm{Pt}(111)$ [57]. A reduction in the number of $3 d$ holes at the Co sites was already detected in the pre-edge of the Co $K$ edge XANES as the negative area $A_{\mathrm{I}}$ (Fig. 4). However, other values of $n_{h}$, as those reported for bulk CoPd alloy and $\mathrm{Au} / \mathrm{CoPd} / \mathrm{Au}$ thin films by DFT calculations $\left(n_{h}=2.60\right)$ [58], or for $\mathrm{Co}_{0.5} \mathrm{Pt}_{0.5} \mathrm{~L}_{0}$ alloy films $\left(n_{h}=2.628\right)$ [59], can lead to a dispersion in the reported values of magnetic moments in alloyed $\mathrm{CoPd}$, which has to be taken into account for comparisons.

Using $n_{h}=2.3$ we found $m_{\mathrm{L}}=0.40(2) \mu_{B}, \quad m_{\mathrm{S}}=$ $1.37(2) \mu_{B}$, and a total magnetic moment $m_{\mathrm{T}}=1.77(4) \mu_{B}$. Table IV collects these values along with those of various Co systems for later comparison.

\section{Pd $L_{2,3}$ edges}

The contribution of $\mathrm{Pd}$ to the total magnetization was studied by XMCD at the Pd $L_{2,3}$ edges. The XAS and corresponding XMCD spectra for the sample with $t_{\mathrm{Pd}}=$ $1.5 \mathrm{~nm}$ are shown in Fig. 13. The nonzero XMCD signal reflects the polarization of the Pd moments by those of Co, and its sign at the $\mathrm{Pd} L_{3}$ edge indicates that $\mathrm{Pd}$ moment is parallel to that of $\mathrm{Co}$, i.e., $\mathrm{Co}$ and $\mathrm{Pd}$ are ferromagnetically coupled. From the sum rules, a ratio $m_{\mathrm{L}} / m_{\mathrm{S}}=-0.006(3)$ was obtained.

The number of holes in the $\mathrm{Pd} 4 d$ band was obtained by comparing the unpolarized XAS intensity at the two $L_{2,3}$ edges of a Pd foil to those of an element with close atomic number, like $\mathrm{Ag}$, recorded under the same experimental conditions, as described in Ref. [67]. Since the absorption per $4 d$ hole, $A_{w l} / n_{h}$, can be written as the difference between the areas subtended by the spectra of the two $n d$ elements in the region of the white line, $A_{w l}^{\mathrm{Pd}}$ and $A_{w l}^{\mathrm{Ag}}$, a value for the absorption $p e r$
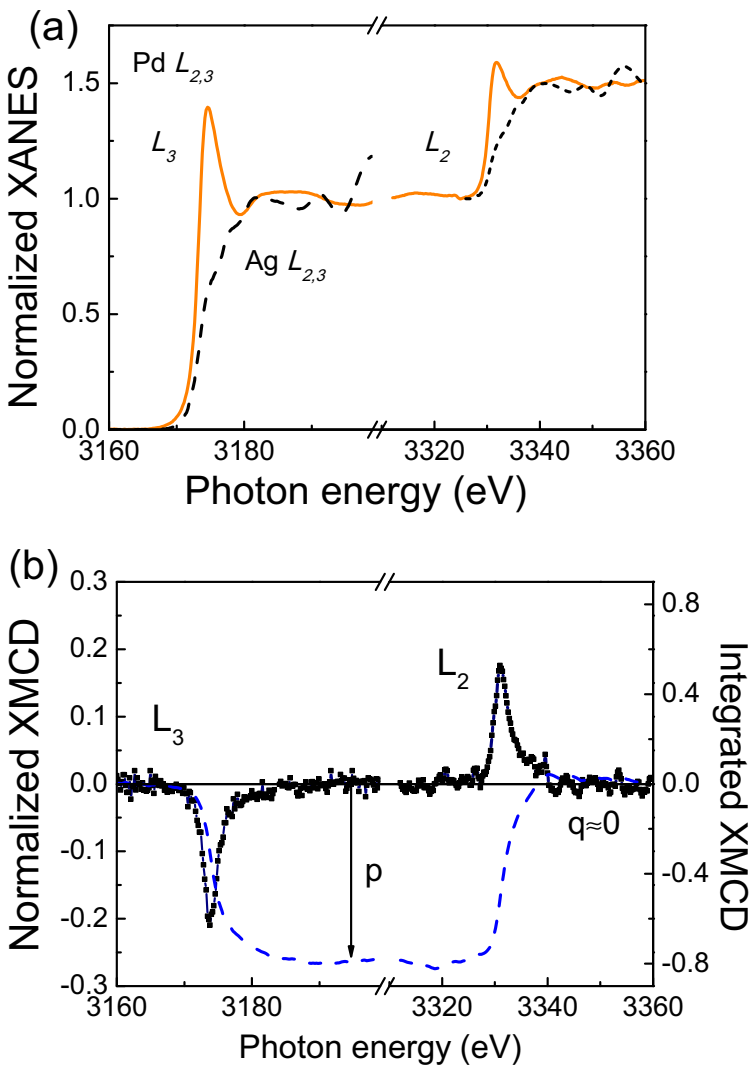

FIG. 13. (a) Normalized XAS and (b) XMCD spectrum at the Pd $L_{2,3}$ edges in the sample with $t_{\mathrm{Co}}=0.7 \mathrm{~nm}$ and $t_{\mathrm{Pd}}=1.5 \mathrm{~nm}$. The XAS spectrum of $\operatorname{Ag} L_{2,3}$ edges is shown in (a) for comparison (dashed line). In (b), the XMCD integrated area is plotted as a dashed line and the end energy limits for the integrals $p$ and $q$ are marked by arrows. 
TABLE IV. Magnetic moments ( $\mu_{B} /$ atom) of Co and Pd obtained from XMCD at the Co and $\mathrm{Pd} L_{2,3}$ edges for various Co-Pd systems. Layer thicknesses are given in $\mathrm{nm}$.

\begin{tabular}{|c|c|c|c|c|c|c|c|c|c|c|}
\hline Multilayer or compound & $m_{\mathrm{L}}^{\mathrm{Co}}$ & $m_{\mathrm{S}}^{\mathrm{Co}}$ & $m_{\mathrm{T}}^{\mathrm{Co}}$ & $m_{\mathrm{L}}^{\mathrm{Co}} / m_{\mathrm{S}}^{\mathrm{Co}}$ & $n_{h}^{\mathrm{Co}}$ & $m_{\mathrm{L}}^{\mathrm{Pd}}$ & $m_{\mathrm{S}}^{\mathrm{Pd}}$ & $m_{\mathrm{T}}^{\mathrm{Pd}}$ & $m_{\mathrm{L}}^{\mathrm{Pd}} / m_{\mathrm{S}}^{\mathrm{Pd}}$ & $n_{h}^{\mathrm{Pd}}$ \\
\hline$\left[3 \mathrm{Al}_{2} \mathrm{O}_{3} / 0.7 \mathrm{Co} / 1.5 \mathrm{Pd}\right]_{25},{ }^{\mathrm{a}}$ & $0.40(2)$ & $1.37(2)$ & $1.77(4)$ & $0.29(1)$ & $2.3(2)$ & $-0.003(2)$ & $0.45(2)$ & $0.45(4)$ & $-0.006(3)$ & $0.85(5)$ \\
\hline$\left[3 \mathrm{Al}_{2} \mathrm{O}_{3} / 0.7 \mathrm{Co} / 0.6 \mathrm{Pd}\right]_{25},[60]$ & 0.24 & 1.64 & 1.88 & 0.15 & 2.3 & & & & & \\
\hline $\mathrm{Co}_{0.5} \mathrm{Pd}_{0.5} \mathrm{NPs}$ on $\mathrm{Au}(111)[58]$ & $0.44^{\mathrm{b}}$ & 1.7 & & 0.26 & 2.60 & & & $\lesssim 0.1$ & & \\
\hline CoPd NPs in C nanotubes [61] & & & & $0.12(5)$ & & & & & $0.07(5)$ & \\
\hline $\mathrm{Co} / \mathrm{Pd}$ interface $[14]^{, \mathrm{c}}$ & $\approx 0.5$ & $\approx 1.9$ & & $\approx 0.28$ & $\approx 3.1$ & & & & & \\
\hline $\mathrm{Co} / \mathrm{Pd}$ center of Co layer [14], c & $\approx 0.15$ & $\approx 1.8$ & & $\approx 0.08$ & $\approx 2.3$ & & & & & \\
\hline$[0.4 \mathrm{Co} / 1 \mathrm{Pd}]_{11},[62]$ & 0.24 & 1.1 & & 0.22 & 2 & & & & & \\
\hline$[0.2-3.2 \mathrm{Co} / 0.8 \mathrm{Pd}]_{N},[63]$ & & & & & & $\sim 0$ & 0.16 & 0.16 & & \\
\hline $\mathrm{Co}_{0.5} \mathrm{Pd}_{0.5} 40 \mathrm{~nm}$-thick films [58] & & & & & & & & 0.4 & & \\
\hline $\mathrm{Co}_{58} \mathrm{Pd}_{42}$ thin films [5] & & & & & & & & 0.44 & 0.098 & \\
\hline Bulk $\mathrm{Co}_{76.9} \mathrm{Pd}_{23.1},[64]$ & & & & & & -0.004 & 0.372 & 0.37 & -0.011 & 1 \\
\hline Bulk CoPd [65] & & & 1.97 & & & & & 0.35 & & \\
\hline Bulk L1 $1_{0} \operatorname{CoPd}(\mathrm{DFT})^{\mathrm{a}}$ & $0.22(4)$ & $1.91(5)$ & $2.1(1)$ & $0.11(2)$ & $2.3(2)$ & $0.06(2)$ & $0.37(4)$ & $0.43(6)$ & $0.16(8)$ & $0.93(3)$ \\
\hline Bulk CoPd (DFT) [58] & & 1.96 & & & 2.59 & & 0.25 & & & 1.10 \\
\hline $\mathrm{Au} / \mathrm{CoPd} / \mathrm{Au}$ films (DFT) [58] & & 2.32 & & & 2.62 & & 0.09 & & & 1.08 \\
\hline$\left[3 \mathrm{Al}_{2} \mathrm{O}_{3} / 0.7 \mathrm{Co} / 1.5 \mathrm{Pt}\right]_{25},[21]$ & $0.20-0.21$ & $1.27-1.34$ & & $0.16(1)$ & & & & $0.2-0.25^{\mathrm{d}}$ & & \\
\hline Co bare NPs $[19,20]$ & $0.21(2)$ & $1.62(2)$ & & $0.13(1)$ & & & & & & \\
\hline Co (hcp) [66] & 0.153 & 1.55 & 1.70 & 0.099 & 2.49 & & & & & \\
\hline $\mathrm{Pd} / \mathrm{Fe}$ multilayers [67] & & & & & & 0.04 & 0.34 & 0.38 & 0.125 & \\
\hline
\end{tabular}

a This work.

${ }^{\mathrm{b}}$ Perpendicular magnetic moment.

${ }^{\mathrm{c}}$ From Fig. 1.

${ }^{\mathrm{d}} m_{T}^{\mathrm{Pt}}$

$4 d$ hole can be derived as

$$
\frac{A_{w l}}{n_{h}}=\frac{A_{w l}^{\mathrm{Pd}}-A_{w l}^{\mathrm{Ag}}}{n_{h}^{\mathrm{Pd}}-n_{h}^{\mathrm{Ag}}} .
$$

Taking for $n_{h}^{\mathrm{Pd}}-n_{h}^{\mathrm{Ag}}=0.92$ [67], $\left(n_{h}^{\mathrm{Pd}}=1.27\right.$ [68], and $n_{h}^{\mathrm{Ag}}=$ 0.35 [69]), and the experimental XAS spectra of a Pd film and $\mathrm{Ag}$, we estimated $A_{w l} / n_{h}=5.18(4) \mathrm{eV} /$ holes, whereby $n_{h}=0.85(5)$ in $\mathrm{Pd}$, which we assume to be also valid for the CoPd NPs. The smaller number of holes in CoPd than in metallic $\mathrm{Pd}\left(n_{h}^{\mathrm{Pd}}=1.27\right)$ reflects the hybridization of the $4 d$ band in $\mathrm{Pd}$ with the $3 d$ band in Co.

The XMCD signals at the $\operatorname{Pd} L_{3}$ and $L_{2}$ edges for the CoPd NPs with $t_{P d}=1.5 \mathrm{~nm}$ show about the same absolute value and opposite signs; therefore the orbital moment is expected to be small. Indeed, an orbital moment $m_{\mathrm{L}}=-0.003(2) \mu_{B}$ was obtained from the sum rules and the $n_{h}$ derived above, while the spin moment is $m_{\mathrm{S}}=0.45(4) \mu_{B}$. The magnetic moments derived from the $\operatorname{Pd} L_{2,3}$ edge XMCD together with those of other CoPd systems are listed in Table IV.

\section{DISCUSSION}

\section{A. Morphology and structure}

The Co-Pd system forms a continuous solid solution, crystallizing with structures based in the face centered cubic structure. Although no superlattice structure has been found, $\mathrm{CoPd}_{3}$ and CoPd can be found as chemically ordered with the $\mathrm{L1}_{2}\left(\mathrm{Cu}_{3} \mathrm{Au}\right.$ type) and the $\mathrm{L}_{0}(\mathrm{CuAu}-\mathrm{I}$ type) structures, respectively, while the Co-rich alloys display an fcc disordered phase, $A_{1}$ [44]. However, short-range order has been reported in $\mathrm{CoPd}_{3}$ and $\mathrm{CoPd}$ as observed in magnetic diffuse neutron scattering $[65,70]$. When prepared as films under appropriate temperature conditions, $\mathrm{Co}_{1-x} \mathrm{Pd}_{x}$ alloy films and $\mathrm{Co} / \mathrm{Pd}$ multilayers have been obtained by a variety of deposition methods and with a large range of $\mathrm{Co}$ and $\mathrm{Pd}$ nominal thicknesses $[10,71,72]$.

The $\left[\mathrm{Al}_{2} \mathrm{O}_{3} / \mathrm{Co} / \mathrm{Pd}\right]_{25}$ multilayers display a granular morphology of self-organized quasi-spherical particles across the layers with average diameter of $\approx 3 \mathrm{~nm}$. This morphology is similar to that of Co-Pt NPs embedded in alumina [21]. Both Co and Pd are present within the particles, as confirmed by EDS and EELS analysis, and increasing the amount of deposited $\mathrm{Pd}$ leads to an excess of metallic $\mathrm{Pd}$, which fills the interparticle spaces and sets up an electrical connection between the NPs. The FFT of HRTEM images and the XRD patterns show the formation of $\mathrm{Co}_{1-x} \mathrm{Pd}_{x}$ alloy with $x \approx 0.5$, grown with the (111) plane parallel to the layer plane (Figs. 1 and 2). XRD also revealed fcc Co and polycrystalline $\mathrm{Pd}$ in the samples with Pd deposited thickness above $0.6 \mathrm{~nm}$, which was found to be highly textured.

For amounts of deposited Pd above $0.6 \mathrm{~nm}$, the shape of the FT magnitude of the Co $K$ edge EXAFS signal resembles that of the $\mathrm{Co}_{50} \mathrm{Pd}_{50}$ bulk alloy (Fig. 5). Likewise, a similar behavior is observed in the FT at the EXAFS signal of the Pd $K$ edge. However, as discussed in Sec. III C 1, a core-shell structure might have been formed, at least in the samples with the lowest Pd deposited thickness. Moreover, although a tetragonal phase was not observed in the XRD patterns, the EXAFS spectra analysis provides evidence of the tetragonal $\mathrm{L}_{0}$ phase. This apparent contradiction can be resolved by 
short-range order: EXAFS, as a local probe, detects shortrange order of $\mathrm{L1}_{0}$-type in small regions, dominant over disordered $\mathrm{A}_{1}$-type regions, while XRD shows an average cubic crystalline CoPd phase in the NPs.

The Co-Co and Co-Pd distances are slightly shorter than those reported for CoPt NPs [21,39], as expected for the smaller metallic radius of $\mathrm{Pd}$. However, although the coordination numbers $N_{\mathrm{Co}-\mathrm{Pd}}$ are similar to those of the CoPt NPs, $N_{\text {Co-Co }}$ are definitely smaller. This may be due to a smaller Co core in the proposed core-shell structure in the current $\mathrm{Co}-\mathrm{Pd}$ NPs, i.e., a deeper alloying in the NPs.

\section{B. Magnetic anisotropy}

Three magnetic phases can be identified, as shown in the magnetic $(H, T)$ phase diagram in Fig. 10(b), namely:

(i) $T<T_{1}$. Unlike the case of $\mathrm{M}=\mathrm{W}, \mathrm{Cu}, \mathrm{Ag}$, and $\mathrm{Au}$ as capping metals, or no capping, the $\left[\mathrm{Al}_{2} \mathrm{O}_{3} / 0.7 \mathrm{~nm} \mathrm{Co} / t_{\mathrm{M}} \mathrm{M}\right]_{25}$ multilayers show ferromagnetic order for $\mathrm{M}=\mathrm{Pd}$ and $\mathrm{Pt}$ at low temperature, which has to be attributed to interparticle interactions. The square shape of the $M_{\perp}(H)$ hysteresis curves [e.g., see Fig. 9(a)] shows strong PMA anisotropy for $T<T_{1}$. The effective anisotropy energy may contain terms associated to the CoPd nanoparticles and the interparticle interaction. $K_{\text {eff }} t$ ranges from 11 to $75 \mathrm{erg} / \mathrm{cm}^{2}$, or $K_{\text {eff }}$ from $0.6 \times 10^{7}$ to $3 \times 10^{7} \mathrm{erg} / \mathrm{cm}^{3}$. These values are similar to or slightly larger than those of all metallic $\mathrm{Co} / \mathrm{Pd}$ films $(1.2-1.4 \times$ $10^{7} \mathrm{erg} / \mathrm{cm}^{3}$ in $[\mathrm{Co}(1 \mathrm{ML}) / \mathrm{Pd}(3 \mathrm{ML})]_{13}$ multilayers [73] and $0.5 \times 10^{7} \mathrm{erg} / \mathrm{cm}^{3}$ in $\mathrm{Co}_{0.5} \mathrm{Pd}_{0.5}$ films [11]), and they show a different dependence with the nonmagnetic element thickness than in the $\mathrm{Al}_{2} \mathrm{O}_{3} / \mathrm{Co} / \mathrm{Pt}$ multilayers [21]. Indeed, while the general trend of $K_{\text {eff }}$ is to decrease with increasing $t_{\mathrm{Pt}}$, this trend is the opposite in the $\mathrm{Al}_{2} \mathrm{O}_{3} / \mathrm{Co} / \mathrm{Pd}$ multilayers, and higher values of $K_{\text {eff }}$ are attained. Moreover, the larger MAE in the CoPd NPs than in the CoPt NPs is concomitant of larger coercive fields and transition temperatures $T_{1}$ (Table III above and Table III in Ref. [21]).

(ii) $T_{1}<T<T_{f}$. Coercivity is zero. However, the $M_{\perp}(H)$ loops in the perpendicular configuration display a sharp drop in the magnetization for vanishing magnetic field, while the decrease in the $M_{\|}(H)$ loops is monotonous [Fig. 9(b)]. A similar behavior has been observed in $\mathrm{Co} / \mathrm{Pd}$ multilayers with comparable Co and Pd thickness but no alumina spacer [13]. The shape of the hysteresis loops is that of a soft ferromagnet with PMA, and it is similar to that of an asperomagnetic phase as observed in amorphous bimetallic systems [74], in which the easy magnetization direction, EMD, varies randomly in a spherical sector, and the spins align along the EMDs. In the CoPd NPs system, a random distribution of EMDs can be produced by misalignment of the NPs growth crystallographic axes in the crystalline particles with respect to the perpendicular to the sample's plane. The increase in the bias field allows the polarization of all moments along the applied field direction and the asperomagnetic-like phase tends to disappear at the freezing temperature $T_{f}$. This intermediate behavior remains for increasing temperature till $T_{f}$, where the ZFC-FC curves merge [Figs. 8(a) and 8(b)].

(iii) $T>T_{f}$. The hysteresis curves are identical, irrespective of field orientation [Fig. 9(c)]. The magnetization is dominated by the thermal fluctuations and the behavior becomes that of an isotropic superparamagnet.

The magnetic anisotropy in the Pd-Co systems may include several contributions like broken symmetry at the atoms of the interface where separate $\mathrm{Co}$ and $\mathrm{Pd}$ phases are in contact, magnetoelastic effects associated to alloying of $\mathrm{Co}$ and $\mathrm{Pd}$, and interatomic interactions in an ordered structure, and different studies have been conducted to identify and separate these contributions. We will focus on systems which may be closer to the current granular multilayers with $\mathrm{Co}_{1-x} \mathrm{Pd}_{x}, x \approx 0.5$ or monolayer (ML) systems which may resemble the $\mathrm{L}_{0}$ phase. On one hand, Engel et al. [75] have reported an interface anisotropy of $0.63 \mathrm{erg} / \mathrm{cm}^{2}$, which constitutes $\approx 80 \%$ of the total anisotropy energy in $0.7 \mathrm{~nm} \mathrm{Co} / \mathrm{Pd}$ multilayers grown along the (111) direction; this is larger than the anisotropy energy per interface currently found for the lower Pd thickness $\left(K_{\text {eff }} t /(2 \times 25)\right)$. On the other hand, Carrey et al. [76] have estimated the magnetoelastic anisotropy in the range $2.3-3.5 \times 10^{6} \mathrm{erg} / \mathrm{cm}^{3}$. The anisotropic magnetostriction constants have been found to be large and very dependent on composition in Co-Pd alloys $[11,77,78]$; in particular, the constant $\lambda_{111}$ of (111)-oriented $\mathrm{Co}_{1-x} \mathrm{Pd}_{x}$ films, $x \approx 0.5$, is large and negative, while $\lambda_{100}$ is positive, which will induce PMA. In the present $\mathrm{Al}_{2} \mathrm{O}_{3} / \mathrm{Co} / \mathrm{Pd}$ multilayers, $\mathrm{Co}_{1-x} \mathrm{Pd}_{x}$ with $x \approx 0.5$, the alloy is found to be oriented with the (111) plane parallel to the layer plane (Figs. 1 and 2). Moreover, the large Debye-Waller factor $\sigma^{2}$ in the Co-Pd distance (Table I) could be produced by structural strain, similarly to reported for CoPt clusters [39]. However, the contribution to MAE of magnetoelastic effects in all-metallic $\mathrm{Co} / \mathrm{Pd}$ multilayers [76] are lower than the anisotropy energy found in the present $\mathrm{Al}_{2} \mathrm{O}_{3} / \mathrm{Co} / \mathrm{Pd}$ multilayers $\left(\geqslant 6 \times 10^{6} \mathrm{erg} / \mathrm{cm}^{3}\right)$.

Solovyev et al. [25] and Kyuno et al. [79] have calculated PMA in bimetallic ordered TX systems $(\mathrm{T}=3 d$ transition metal, $\mathrm{X}=$ heavy metal), in particular in CoPd and CoPt systems, using a perturbative treatment of the spin-orbit interaction, on a $\mathrm{L}_{0}$ structure modelled as a finite number of alternating $\mathrm{Co}$ and $\mathrm{Pd}$ layers, and on monolayers of $\mathrm{Co}$ separated by $2 \mathrm{ML}$ of $\mathrm{Pd}$, respectively. Under this approximation, the interatomic contributions to the magnetocrystalline anisotropy at a given site is quadratic with the spin-orbit constants. The calculated MAE for CoPd amounts to $1.0 \times$ $10^{-5}$ Ry [25], yielding to a value of $K_{\text {eff }} \approx 1.9 \times 10^{7} \mathrm{erg} / \mathrm{cm}^{3}$ (the fct unit cell of Ref. [35] was used), which is close to the current result for $t_{\mathrm{Pd}}=1.5 \mathrm{~nm}$. Similarly, Kyuno et al. [79] provides a MAE value of $1.12 \mathrm{meV} / \mathrm{unit}$ cell for a multilayer structure of Co monolayers separated by two Pd monolayers, which corresponds to $\approx 1.4 \times 10^{7} \mathrm{erg} / \mathrm{cm}^{3}$ (unit cell as defined in Ref. [80]), while Ye et al. [81] obtain a MAE of $\approx 0.5 \times 10^{7} \mathrm{erg} / \mathrm{cm}^{3}$ for bulk $\mathrm{L1}_{0}$. The calculation of the contribution of the different layers [25] shows that the magnetic anisotropy is perpendicular and mainly originated at the Co site by the atoms at the same atomic layer, while the total contribution at the Pd sites is small and parallel to the layers plane. Then, Co-Pd hybridization is the main contribution to MAE in the current CoPd NPs with the lower Pd deposited thickness, and other sources like magnetoelasticity constitute minor contributions. However, for the larger Pd thickness (Table III), at least three times large $K_{\text {eff }}$ values are found and additional sources of anisotropy should be sought. 
Although the case of the CoPt NPs on $\mathrm{Al}_{2} \mathrm{O}_{3}$ should be expected to be similar to the Pd counterpart, there are important differences. The same calculation mentioned above [25] yields to a much higher MAE in that case, since it is quadratic with the spin-orbit constant and $\left(\xi_{\mathrm{Pt}} / \xi_{\mathrm{Pd}}\right)^{2} \approx 9$; however, its MAE has been experimentally determined to be an order of magnitude smaller, and it decreases with the amount of deposited $\mathrm{Pt}$ [21].

In the series of multilayers $\left[\mathrm{Al}_{2} \mathrm{O}_{3} / \mathrm{Co} / \mathrm{M}\right]_{N}$ with $\mathrm{M}$ a transition metal, ferromagnetic behavior has been observed only for Pd and Pt cappings up to date [21], in contrast to uncapped Co layers or layers capped with $\mathrm{W}[22]$, or noble metals $(\mathrm{Cu}$, $\mathrm{Ag}$, and $\mathrm{Au}$ ) [19,20]. The intralayer interparticle magnetic interaction within the CoPt layers in the $\left[\mathrm{Al}_{2} \mathrm{O}_{3} / \mathrm{Co} / \mathrm{Pt}\right]_{N}$ multilayers has been suggested to be of RKKY type via the $\mathrm{Pt}$ atoms [21], in a similar way to the interlayer coupling in all-metallic $\mathrm{Co} / \mathrm{Pt}$ multilayers [16]. It is important to notice that Knepper \& Yang [16] have suggested that this RKKY interaction would couple polarized $\mathrm{Pt}$ atoms at the interface of adjacent Co layers. Interestingly, in the $\left[\mathrm{Al}_{2} \mathrm{O}_{3} / \mathrm{Co} / \mathrm{M}\right]_{N}$ multilayers, ferromagnetic order is only observed when the $\mathrm{M}$ metal is highly polarized, i.e., the cases of $\mathrm{Pt}$ and $\mathrm{Pd}$. Therefore, it may be proposed that RKKY is responsible for the intralayer FM coupling in the current multilayer system with either Pt or $\mathrm{Pd}$ as a consequence of hybridization induced polarization of the capping atoms. The higher polarization encountered in the Pd multilayers would account for the slightly higher ordering temperature $T_{f}$. Unfortunately, the oscillatory behavior of the RKKY interaction cannot be observed because the distance between the CoPd nanoparticles cannot be controlled. It is worth noticing that the polarization of Pd atoms is present even in the sample with only $0.6 \mathrm{~nm}$ of $\mathrm{Pd}$ [60], and $T_{f}$ is similar to that in the samples with more deposited Pd (Table III); therefore the interparticle link for RKKY interaction has formed although alloying with Co is not as complete as in the other samples.

Focusing on the comparison between the Pd and Pt cases with the same thickness, it may be observed that while the ordering temperature $T_{f}$ is similar in both cases, the temperature $T_{1}$ is approximately constant in the $\mathrm{Pt}$ case, but it is larger and with an increasing general trend for $\mathrm{Pd}$ ( $K_{\text {eff }}$ in Table III). Since $T_{1}$ is related to the development of coercivity, it is associated to the higher MAE in the CoPd multilayers. The dependence on the capping metal deposited thickness is also different. While the magnetic anisotropy in the Pt multilayers shows an overall decrease with the Pt deposited thickness, the general trend in the Pd multilayers is an increase. However, in either case the MAE constant $K_{\text {eff }} t$ is at least $\approx 10 \mathrm{erg} / \mathrm{cm}^{2}$, definitely higher than the anisotropy energies calculated on $\mathrm{Pd} / \mathrm{Co}$ layered structures [25,79,81]. This suggests that interparticle interactions may also contribute to the magnetic anisotropy.

However, in contrast to this anisotropy requirement, the standard RKKY theory is isotropic, since it describes the interaction of two localized spin moments set up by conduction electrons as proportional to $\vec{S}_{1} \cdot \vec{S}_{2}$, where $\vec{S}_{1}$ and $\vec{S}_{2}$ are the localized moments. The inconsistency with respect to magnetic anisotropy also holds for the dipolar interaction. Anisotropy due to interaction between well separated moments has been proposed in spin glasses to arise from an additional term of Dzyaloshinskii-Moriya type, $\propto \vec{S}_{1} \times \vec{S}_{2}$, produced by intermediate spin-orbit scattering by nonmagnetic impurities and host atoms [82]. Alternatively, Staunton et al. [83] have produced both the isotropic standard RKKY term and a Dzyaloshinskii-Moriya type term plus a pseudodipolar term from a relativistic generalization of the RKKY interaction where spin orbit is treated nonperturbatively. The obtained Dzyaloshinskii-Moriya type term is

$$
J_{\mathrm{DM}} \propto\left(\vec{S}_{1} \cdot \vec{S}_{2}\right)\left[\vec{R}_{12} \cdot\left(\vec{S}_{1} \times \vec{S}_{2}\right)\right]^{2}
$$

where $R_{12}$ is the distance between the two spin moments. As shown by Staunton et al. for polarized $\mathrm{Pt}$, this term yields to an anisotropy energy which favors parallel alignment of spins perpendicular to the interspin line, i.e., PMA in the multilayer systems, which amounts to an additional term to the intraparticle magnetic anisotropy associated to the Co-Pd hybridization with the $\mathrm{L} 1_{0}$ structure that could explain the higher PMA in the present compounds.

\section{Magnetic moments}

The saturation magnetization measured by SQUID magnetometry shows a sharp increase from $1.9 \times 10^{-3} \mathrm{emu} / \mathrm{cm}^{2}$ for $t_{\mathrm{Pd}}=0.6$ and $1.5 \mathrm{~nm}$ to $\approx 3.3 \times 10^{-3} \mathrm{emu} / \mathrm{cm}^{2}$ for $t_{\mathrm{Pd}}=4.5$ and $6.0 \mathrm{~nm}$ (Table III). Such a discontinuity in $M_{S}$ has not been observed in the Pt case [21]. Alternatively, $M_{S}$ can be derived from the total magnetic moment of $\mathrm{Co}$ and $\mathrm{Pd}$ atoms from the XMCD data of the $t_{\mathrm{Pd}}=1.5 \mathrm{~nm}$ sample. For Co, $m_{T}^{\text {Co }}=$ $1.77(4) \mu_{B}$, which using the nominal thickness of deposited Co $\left(t_{\mathrm{Co}}=0.7 \mathrm{~nm}\right)$ yields $M_{S}=2.6(1) \times 10^{-3} \mathrm{emu} / \mathrm{cm}^{2}$. Including the contribution of $\mathrm{Pd}\left(m_{T}^{\mathrm{Pd}}=0.45(4) \mu_{B}\right)$ is not straightforward, since the amount of polarized Pd is unknown. For Pt and Pd the extent of polarization by the Co atoms is up to about 5 atomic layers or $\approx 1 \mathrm{~nm}[84,85]$. As an estimation, the polarization of $0.6 \mathrm{~nm}$ of $\mathrm{Pd}$, corresponding to a sample for which it can be assumed that all Pd is alloyed within the Co NPs, will add $\approx 0.4 \times 10^{-3} \mathrm{emu} / \mathrm{cm}^{2}$. The difference in the values of $M_{S}$ from SQUID and XMCD experiments cannot be explained by a different choice in the value of the number of $3 d$ holes used in the calculation of Co magnetic moments from XMCD data, since this would require unphysical low values of $n_{h}$.

It must be recalled that total electron yield XMCD at the Co $L_{2,3}$ edges will only probe the uppermost layer of the sample [55]. Then, the reduced value of the macroscopic $M_{S}$ with respect to the XMCD "single-layer" estimation might suggest an interlayer exchange coupling, IEC, where magnetic layers are not all coupled parallel. Similarly, from recent XMCD results for the $t_{\mathrm{Pd}}=0.6 \mathrm{~nm}$ sample [60] a saturation magnetization value $M_{S}=2.8 \times 10^{-3} \mathrm{emu} / \mathrm{cm}^{2}$ has been derived (only the Co contribution), again larger than the measured macroscopic value of $1.9 \times 10^{-3} \mathrm{emu} / \mathrm{cm}^{2}$. However, IEC through insulating spacers is known to decrease exponentially with the spacer thickness $[86,87]$, and it has not been observed in amorphous insulating spacers like $\mathrm{Al}_{2} \mathrm{O}_{3}$ [88,89]. Therefore, in the $\mathrm{Al}_{2} \mathrm{O}_{3} / \mathrm{Co} / \mathrm{Pd}$ multilayers with $3 \mathrm{~nm}$ spacers the IEC should not be expected. In contrast, IEC has been observed in the all-metallic $[\mathrm{Co} / \mathrm{Pd}]_{N}$ multilayers, and this has been attributed to RKKY interactions [16]. 
The ratio of the orbital-to-spin moments reported for the present CoPd NPs in the $\left[\mathrm{Al}_{2} \mathrm{O}_{3} / \mathrm{Co} / \mathrm{Pd}\right]_{n}$ multilayers is larger than that in bulk fcc Co $\left(m_{\mathrm{L}} / m_{\mathrm{S}}=0.078\right)$ [90] and bare Co NPs in the same type of multilayers $\left(m_{\mathrm{L}} / m_{\mathrm{S}}=0.13\right)$ [20]. The clear enhancement, by a factor of 3 , is due to the increase in the Co $3 d$ orbital magnetic moment, and the decrease in the spin magnetic moment, as a consequence of hybridization with $\mathrm{Pd}$. Interestingly, in the Pt counterpart of these multilayers [21] the spin moment is similar, but the orbital moment is a half in spite of the larger spin-orbit interaction in $\mathrm{Pt}$, which shows the pre-eminence of hybridization in the development of the orbital moment at the Pd atoms.

Similar high values of the orbital moment have been found in $\mathrm{Co}_{0.5} \mathrm{Pd}_{0.5}$ nanoclusters (i.e., composition similar to that encountered currently) on $\mathrm{Au}(111)$ [58] and at the $\mathrm{Co} / \mathrm{Pd}$ interface in $\mathrm{Pd} / \mathrm{Co} / \mathrm{Pd}$ trilayer [14]. However, it must be noticed that in these two systems the number of $3 d$ holes used to derive the magnetic moments from the XMCD data is rather high: 2.6 and 3.1, respectively. In particular, in Ref. [14], it is shown that $n_{h}$ decreases with the distance to the $\mathrm{Co} / \mathrm{Pd}$ interface and at the centre of the Co layer $n_{h} \approx 2.4$ and $m_{\mathrm{L}} \approx 0.18 \mu_{\mathrm{B}}$; the high $m_{\mathrm{L}}$ at the interface is produced by symmetry breaking, while the orbital moment we have currently obtained is produced by $3 d$ Co- $4 d$ Pd hybridization in the $\mathrm{L} 1_{0}$ structure alloy.

The orbital moment of $\mathrm{Pd}$ in the $\left[\mathrm{Al}_{2} \mathrm{O}_{3} / 0.7 \mathrm{~nm} \mathrm{Co} / 1.5 \mathrm{~nm} \mathrm{Pd}\right]_{25}$ multilayer is almost zero, while the spin moment amounts to $0.45 \mu_{B}$. It should be recalled that only the $\mathrm{Pd}$ atoms polarized by Co contribute to the XMCD signal, although all the Pd atoms are contained in the XAS spectrum. Then, the magnetic moments extracted from the XMCD integral values could be lower than the actual ones. Very small Pd orbital moments and the enhancement of the spin moment with respect to pure $\mathrm{Pd}$ ( $m_{\mathrm{S}} \approx 0.012 \mu_{B}$, Ref. [91]) have been systematically found in Co-Pd systems $[5,63,64]$. However, the enhancement in very thin Pd layers, like the [Co/0.8 nm Pd] multilayers [63], or in $0.4 \mathrm{ML} \mathrm{Co}_{0.5} \mathrm{Pd}_{0.5}$ nanoclusters on $\mathrm{Au}(111)$ [58], is at least two times smaller than in $\mathrm{Co}_{1-x} \mathrm{Pd}_{x}$ much thicker films [5,58,64] or bulk alloys (Table IV) [65,92]. The low $\mathrm{Pd}$ moment in the $\mathrm{Co}_{0.5} \mathrm{Pd}_{0.5}$ nanoclusters on $\mathrm{Au}$ has been attributed to the stretching of the alloy to epitaxial conditions on $\mathrm{Au}(111)$ that reduces the $\mathrm{Pd}$ moment. In contrast, the $\mathrm{Pd}$ induced moments in the present $\left[\mathrm{Al}_{2} \mathrm{O}_{3} / \mathrm{Co} / \mathrm{Pd}\right]_{25}$ multilayers are similar to those of the $\mathrm{Co}_{1-x} \mathrm{Pd}_{x}$ alloys, which shows that strain effects are minor with respect that of the chemical vicinity of Co atoms, in agreement with the behavior of the MAE discussed above.

The increased Co orbital moments in $\mathrm{Co} / \mathrm{Pd}$ systems with respect to pure $\mathrm{Co}$ is intrinsic to $\mathrm{Co} / \mathrm{Pd}$ layered structures. Indeed, calculations in the single-impurity approximation for the spin-orbit interaction in the $\mathrm{L} 1_{0}$ structure [25], i.e., the spin-orbit interaction is switched off at all sites except at the considered $\mathrm{Co}$ or $\mathrm{Pd}$ atom in the structure, show that the single-site contribution to the orbital magnetic moment is dominant and the orbital to spin ratio is $m_{\mathrm{L}}^{\mathrm{Co}} / m_{\mathrm{S}}^{\mathrm{Co}}=0.44$. However, the calculated orbital moment $m_{\mathrm{L}}^{\mathrm{Co}}=0.11 \mu_{B}$ is lower than the experimental values (Table IV). Interestingly, the calculation with spin orbit at every site in a perturbative treatment shows that the orbital magnetic moment of a Co or Pd atom is produced mainly by its layer, since the net contribution

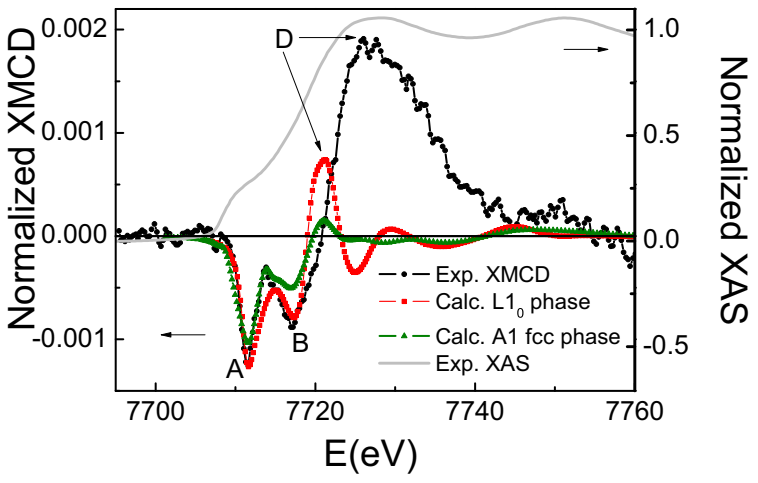

FIG. 14. Experimental XMCD (•) and XAS (solid line) signals at the Co $K$ edge for CoPd NPs. Calculated XMCD signals at the Co $K$ edge of nanoparticles with the $\mathrm{L} 1_{0}(\square)$ and $\mathrm{A} 1(\triangle)$ structures.

of adjacent layers tend to cancel out [25]. However, although the intra- and interlayer contributions hardly modify the single-site $m_{\mathrm{L}}^{\text {Co }}$ value, they decrease $m_{\mathrm{L}}^{\mathrm{Pd}}$, but the predicted value for the latter is not negligible [25], contrary to our observation. Moreover, Kyuno et al. [79] have compared the local density of states (LDOS) of bulk Co, a single Co ML, and a Co MLs separated by two Pd MLs. They show that the unsupported Co ML produces an unquenched orbital moment, but two Pd MLs separating the Co layers are enough to almost retrieve the practically quenched orbital moment of bulk Co. Therefore, the $\mathrm{L}_{0}$ structure allows for the formation of the orbital moment in Co because of its alternating $\mathrm{Co}$ and $\mathrm{Pd}$ MLs.

The effect of chemical structure on the electronic hybridization in the current $\mathrm{Co}-\mathrm{Pd}$ and the previously studied Co-Pt systems can be checked by comparing calculated and experimental $\mathrm{x}$-ray absorption spectra. X-ray absorption cross sections were calculated self-consistently with the fully relativistic FDMNES code [26,27] in the framework of multiple scattering theory within the muffin-tin approximation. The calculations were also utilized to derive hole numbers at the $3 d \mathrm{Co}$ and $4 d \mathrm{Pd}$ orbitals used to produce spin and orbital moments from the XMCD spectra in Sec. IV B. Several cases were simulated: NPs with the tetragonalized chemically ordered $\mathrm{L}_{0}$ phase and the bulk $50 \%-50 \%$ alloys with the $\mathrm{L}_{0}$ and A1 structures (see Supplemental Material [93] for further details). In the CoPd NPs with both the $\mathrm{L}_{0}$ and $\mathrm{A} 1$ structures, peaks $\mathrm{A}$ and $\mathrm{B}$ of the calculated spectrum appear well separated $(\approx 5 \mathrm{eV})$, in agreement with the experimental spectrum (Fig. 14). However, the relative intensity between A and $\mathrm{B}$ peaks is better described by the $\mathrm{L}_{0}$ theoretical curve. In contrast, the separation in the CoPt NPs case is $\approx 3 \mathrm{eV}$ in the calculated spectrum for the $\mathrm{L}_{0}$ phase, both peaks coalesce for the A1 phase, and the experimental separation is $\approx 10 \mathrm{eV}$ (see Fig. 16 in Ref. [21]). At higher energies, the calculated peaks are strongly reduced in intensity in the $\mathrm{L} 1_{0}$ curve, while practically absent in the A1 one. This is expected for the sum of the oscillating XMCD from every Co atom, slightly shifted in energy by local chemical disorder. Moreover, the A and B minima are predicted in the calculated spectra for both bulk $\mathrm{CoPd}$ and $\mathrm{CoPt}$, ordered in the $\mathrm{L} 1_{0}$ phase, with a intensity ratio $r=I_{A} / I_{B}>1$ in the CoPd case, while $r<1$ for the CoPt, 
in good agreement with experiment (Fig. 3 in Supplemental Material [93]).

\section{CONCLUSIONS}

An extensive study on a family of $\left[\mathrm{Al}_{2} \mathrm{O}_{3} / 0.7 \mathrm{~nm}\right.$ $\left.\mathrm{Co} / t_{\mathrm{Pd}} \mathrm{Pd}\right]_{25}$ multilayers, obtained by room temperature sequential sputtering deposition, has been presented. From XRD, electron microscopy and EXAFS, the morphology of the multilayers is deduced to consists in self-organized bimetallic CoPd nanoparticles with chemical order compatible with short-order in the $\mathrm{L}_{0}$ structure and a small pure Co core. Above $\mathrm{Pd}$ deposition thickness of $\sim 1 \mathrm{~nm}$, Pd-Co alloying is complete and the additional Pd fills the interparticle space. The collective magnetic behavior of the CoPd NPs depends on temperature, and three magnetic phases were identified: ferromagnetic with coercive field below $T_{1}$; soft ferromagnetic in the region $T_{1}<$ $T<T_{f}$, with a behavior similar of that of an asperomagnet; and superparamagnetic behavior above $T_{f}$. In this paper we report $T_{1}$ as large as $350 \mathrm{~K}$ for the sample with $t_{\mathrm{Pd}}=6 \mathrm{~nm}$.

These multilayers show perpendicular magnetic anisotropy at low temperature with effective anisotropy constant $K_{\text {eff }} t$ of at least $0.4 \mathrm{erg} / \mathrm{cm}^{2}$ per layer, which is directly related to the short range order of the $\mathrm{Co}_{50} \mathrm{Pd}_{50} \mathrm{L1}_{0}$-like phase. The observed anisotropy constant is larger than those of bulk Co, multilayers of bare Co NPs [20], and other bimetallic multilayers with alloyed NPs like CoW [22] and CoPt, [21] in contradiction with previous calculations [25]. XANES at the $\mathrm{Co} K$ and $\mathrm{Pd} K$ edges proves the existence of Co-Pd hybridization, which has also been confirmed by comparison of experimental XMCD results at the Co $K$ edge with simulations. The XMCD spectra at the Co $L_{2,3}$ edges show an important enhancement of the Co orbital moments. Moreover, the XMCD spectra at the $\operatorname{Pd} L_{2,3}$ edges demonstrate the polarization of $\mathrm{Pd}$ atoms reflected on its spin moment, while the orbital moment is almost quenched. Both effects are related to an $\mathrm{L1}_{0}$-type structure at the CoPd nanoparticles with $a \approx 50 \%$ alloying stoichiometry.

The ferromagnetic behavior of the multilayers is attributed to RKKY interaction within each layer between polarized $\mathrm{Pd}$ through the unpolarized interparticle Pd. Besides, relativistic RKKY is proposed to supply the extra anisotropy for the multilayers with larger Pd thickness, with respect to the calculated intrinsic anisotropy of $\mathrm{L}_{0}$-order type origin. The larger polarization of $\mathrm{Pd}$ with respect to $\mathrm{Pt}$ in the same type of multilayers would then be responsible for the higher ferromagnetic order temperatures and anisotropy constants.

\section{ACKNOWLEDGMENTS}

The financial support of the Spanish Ministerio de Economía MAT2014-53921-R and Aragonese DGA-IMANA E34 projects is acknowledged. Authors would like to acknowledge the use of Servicio General de Apoyo a la Investigación-SAI, Universidad de Zaragoza. European Synchrotron Radiation Facility beamtime corresponds to HE2238, HE2541, HE2952, and HE3136 experiments.
[1] C. T. Sousa, D. C. Leitao, M. P. Proenca, J. Ventura, A. M. Pereira, and J. P. Araujo, Appl. Phys. Rev. 1, 031102 (2014).

[2] C. Castán-Guerrero, J. Herrero-Albillos, J. Bartolomé, F. Bartolomé, L. A. Rodríguez, C. Magén, F. Kronast, P. Gawronski, O. Chubykalo-Fesenko, K. J. Merazzo, P. Vavassori, P. Strichovanec, J. Sesé, and L. M. García, Phys. Rev. B 89, 144405 (2014).

[3] J. B. Haun, T.-J. Yoon, H. Lee, and R. Weissleder, Wiley Interdiscip. Rev.: Nanomed. and Nanobiotechnol. 2, 291 (2010).

[4] H. Zeng, J. Li, J. P. Liu, Z. L. Wang, and S. Sun, Nature (London) 420, 395 (2002).

[5] A. Vlachos, S. D. Pappas, V. Kapaklis, V. Karoutsos, A. Kordatos, F. Wilhelm, A. Rogalev, P. Fumagalli, P. Poulopoulos, M. J. Velgakis, and C. Politis, J. Nanosci. Nanotechnol. 12, 6240 (2012).

[6] L. G. Vivas, R. Yanes, O. Chubykalo-Fesenko, and M. Vázquez, Appl. Phys. Lett. 98, 232507 (2011).

[7] J.-W. Liao, U. Atxitia, R. F. L. Evans, R. W. Chantrell, and C.-H. Lai, Phys. Rev. B 90, 174415 (2014).

[8] A. Ebbing, O. Hellwig, L. Agudo, G. Eggeler, and O. Petracic, Phys. Rev. B 84, 012405 (2011).

[9] F. Aguilera-Granja, A. Vega, J. Rogan, X. Andrade, and G. García, Phys. Rev. B 74, 224405 (2006).

[10] I. K. Schuller, S. Kim, and C. Leighton, J. Magn. Magn. Mater. 200, 571 (1999).

[11] S. Tsunashima, K. Nagase, K. Nakamura, and S. Uchiyama, IEEE Trans. Magn. 25, 3761 (1989).
[12] J. R. Childress, J. L. Duvail, S. Jasmin, A. Barthélémy, A. Fert, A. Schuhl, O. Durand, and P. Galtier, J. Appl. Phys. 75, 6412 (1994).

[13] P. F. Carcia, J. Appl. Phys. 63, 5066 (1988).

[14] S.-K. Kim and J. B. Kortright, Phys. Rev. Lett. 86, 1347 (2001).

[15] S.-K. Kim, V. A. Chernov, J. B. Kortright, and Y. M. Koo, Appl. Phys. Lett. 71, 66 (1997).

[16] J. W. Knepper and F. Y. Yang, Phys. Rev. B 71, 224403 (2005).

[17] J. Briático, J.-L. Maurice, J. Carrey, D. Imhoff, F.Petroff, and A. Vaurès, Eur. Phys. J. D 9, 517 (1999).

[18] J.-L. Maurice, J. Briático, J. Carrey, F. Petroff, L. F. Schelp, and A. Vaurès, Philos. Mag. 79, 2921 (1999).

[19] F. Luis, F. Bartolomé, F. Petroff, J. Bartolomé, L. M. García, C. Deranlot, H. Jaffrès, M. J. Martínez, P. Bencok, F. Wilhelm, A. Rogalev, and N. Brookes, Europhys. Lett. 76, 142 (2006).

[20] J. Bartolomé, L. M. García, F. Bartolomé, F. Luis, R. LópezRuiz, F. Petroff, C. Deranlot, F. Wilhelm, A. Rogalev, P. Bencok, N. B. Brookes, L. Ruiz, and J. M. González-Calbet, Phys. Rev. B 77, 184420 (2008).

[21] A. I. Figueroa, J. Bartolomé, L. M. García, F. Bartolomé, O. Bunău, J. Stankiewicz, L. Ruiz, J. M. González-Calbet, F. Petroff, C. Deranlot, S. Pascarelli, P. Bencok, N. B. Brookes, F. Wilhelm, A. Smekhova, and A. Rogalev, Phys. Rev. B 90, 174421 (2014).

[22] A. I. Figueroa, J. Bartolomé, L. M. García, F. Bartolomé, C. Magén, A. Ibarra, L. Ruiz, J. M. González-Calbet, F. Petroff, C. 
Deranlot, S. Pascarelli, P. Bencok, N. B. Brookes, F. Wilhelm, and A. Rogalev, Phys. Rev. B 84, 184423 (2011).

[23] A. R. Mackintosh and O. K. Andersen, The electronic structure of transition metals, in Electrons at the Fermi surface, edited by M. Springford (Cambridge University Press, Cambridge, 1980), Chap. 5, pp. 149-224.

[24] J. Bartolomé, A. I. Figueroa, F. Bartolomé, L. M. García, F. Wilhem, and A. Rogalev, Solid State Phenom. 194, 92 (2013).

[25] I. V. Solovyev, P. H. Dederichs, and I. Mertig, Phys. Rev. B 52, 13419 (1995).

[26] Y. Joly, Phys. Rev. B 63, 125120 (2001).

[27] O. Bunău and Y. Joly, J. Phys.: Condens. Matter 21, 345501 (2009).

[28] D. Babonneau, F. Petroff, J. L. Maurice, F. Fettar, A. Vaures, and A. Naudons, Appl. Phys. Lett. 76, 2892 (2000).

[29] F. Luis, J. M. Torres, L. M. García, J. Bartolomé, J. Stankiewicz, F. Petroff, F. Fettar, J. L. Maurice, and A. Vaures, Phys. Rev. B 65, 094409 (2002).

[30] See Supplemental Material at http://link.aps.org/supplemental/ 10.1103/PhysRevB.93.174410 for EDS and XRD measurements of bulk alloys reference samples and XRD fits of CoPd multilayers.

[31] J. Rodriguez-Carvajal, Phys. B 192, 55 (1993).

[32] A. L. Patterson, Phys. Rev. 56, 978 (1939).

[33] JCPD Card No 46-1043.

[34] R. M. Bozorth, P. A. Wolff, D. D. Davis, V. B. Compton, and J. H. Wernick, Phys. Rev. 122, 1157 (1961).

[35] J. R. Cerdá, P. L. de André, A. Cebollada, R. Miranda, E. Navas, C. M. Schuster, P. Schneider, and J. Kirschner, J. Phys.: Condens. Matter 5, 2055 (1993).

[36] E. A. Owen and D. M. Jones, Proc. Phys. Soc. B 67, 456 (1954).

[37] JCPD Card No 15-0806.

[38] B. Ravel and M. Newville, J. Synchrotron Radiat. 12, 537 (2005).

[39] N. Blanc, L. E. Díaz-Sánchez, A. Y. Ramos, F. Tournus, H. C. N. Tolentino, M. De Santis, O. Proux, A. Tamion, J. TuaillonCombes, L. Bardotti, O. Boisron, G. M. Pastor, and V. Dupuis, Phys. Rev. B 87, 155412 (2013).

[40] A. I. Figueroa, in Magnetic Nanoparticles. A Study by Synchrotron Radiation and RF Transverse Susceptibility, Springer Theses (Springer International Publishing, Switzerland, 2015), pp. 31-117.

[41] F. Groot and A. Kotani, Core Level Spectroscopy of Solids, Advances in Condensed Matter Science (CRC Press, Taylor \& Francis Group, Boca Raton, 2008).

[42] http://icsd.iqfr.csic.es/icsd/.

[43] B. Mierzwa, J. Alloys Compd. 362, 178 (2004).

[44] Y. Matsuo, J. Phys. Soc. Jpn. 32, 972 (1972).

[45] J. A. McCaulley, Phys. Rev. B 47, 4873 (1993).

[46] J. A. McCaulley, Phys. Rev. B 48, 666 (1993).

[47] C.-M. Lin, T.-L. Hung, Y.-H. Huang, K.-T. Wu, M.-T. Tang, C.-H. Lee, C. T. Chen, and Y. Y. Chen, Phys. Rev. B 75, 125426 (2007).

[48] See Supplemental Material at http://link.aps.org/supplemental/ 10.1103/PhysRevB.93.174410 for Pd $K$ edge EXAFS analysis.

[49] B. Mierzwa, Z. Kaszkur, B. Moraweck, and J. Pielaszek, J. Alloys Compd. 286, 93 (1999).

[50] M. T. Johnson, P. J. H. Bloemen, F. J. A. den Broeder, and J. J. de Vries, Rep. Prog. Phys. 59, 1409 (1996).
[51] In Ref. [21] $K_{\text {eff }}$ of Table III has to be divided by 25 (number of layers) to produce the correct value in $\mathrm{erg} / \mathrm{cm}^{3}$.

[52] B. T. Thole, P. Carra, F. Sette, and G. vanderLaan, Phys. Rev. Lett. 68, 1943 (1992).

[53] P. Carra, B. T. Thole, M. Altarelli, and X. Wang, Phys. Rev. Lett. 70, 694 (1993).

[54] R. Laskowski and P. Blaha, Phys. Rev. B 82, 205104 (2010).

[55] G. van der Laan and A. I. Figueroa, Coor. Chem. Rev. 277-278, 95 (2014).

[56] M. G. Samant, J. Stohr, S. S. P. Parkin, G. A. Held, B. D. Hermsmeier, F. Herman, M. van Schilfgaarde, L.-C. Duda, D. C. Mancini, N. Wassdahl, and R. Nakajima, Phys. Rev. Lett. 72, 1112 (1994).

[57] P. Gambardella, S. Rusponi, M. Veronese, S. S. Dhesi, C. Grazioli, A. Dallmeyer, I. Cabria, R. Zeller, P. H. Dederichs, K. Kern, C. Carbona, and H. Brune, Science 300, 1130 (2003).

[58] P. Ohresser, E. Otero, F. Wilhelm, A. Rogalev, C. Goyhenex, L. Joly, H. Bulou, M. Romeo, V. Speisser, J. Arabski, G. Schull, and F. Scheurer, J. Appl. Phys. 114, 223912 (2013).

[59] W. Grange, I. Galanakis, M. Alouani, M. Maret, J.-P. Kappler, and A. Rogalev, Phys. Rev. B 62, 1157 (2000).

[60] L. G. Vivas, A. Figueroa, F. Bartolomé, J. Rubín, L. García, C. Deranlot, F. Petroff, L. Ruiz, J. M. González-Calbet, N. B. Brookes, F. Wilhelm, A. Rogalev, and J. Bartolomé, J. Magn. Magn. Mater. 400, 248 (2015).

[61] D. B. Roa, I. D. Barcelos, A. de Siervo, K. R. Pirota, R. G. Lacerda, and R. Magalhaes-Paniago, Appl. Phys. Lett. 96, 253114 (2010).

[62] Y. Wu, J. Stohr, B. D. Hermsmeier, M. G. Samant, and D. Weller, Phys. Rev. Lett. 69, 2307 (1992).

[63] H. Sakurai, F. Itoh, Y. Okabe, H. Oike, and H. Hashimoto, J. Magn. Magn. Mater. 198-199, 662 (1999).

[64] K. Kobayashi, H. Maruyama, T. Iwazumi, N. Kawamura, I. Inoue, and H. Yamazaki, J. Electron Spectrosc. Relat. Phenom. 78, 303 (1996).

[65] J. W. Cable, E. O. Wollan, and W. C. Koehler, Phys. Rev. 138 A755 (1965).

[66] C. T. Chen, Y. U. Idzerda, H.-J. Lin, N. V. Smith, G. Meigs, E. Chaban, G. H. Ho, E. Pellegrin, and F. Sette, Phys. Rev. Lett. 75, 152 (1995).

[67] J. Vogel, A. Fontaine, V. Cros, F. Petroff, J.-P. Kappler, G. Krill, A. Rogalev, and J. Goulon, Phys. Rev. B 55, 3663 (1997).

[68] C. A. Kuhnen and E. Z. da Silva, Phys. Rev. B 46, 8915 (1992).

[69] A. Bzowski, T. K. Sham, and Y. M. Yiu, Phys. Rev. B 49, 13776 (1994).

[70] A. A. Katsnelson, S. A. Alimov, P. S. Dazhayev, V. M. Silonov, and N. N. Stupina, Phys. Met. Metallogr. 26, 26 (1968).

[71] D. Weller, H. Brändle, and C. Chappert, J. Magn. Magn. Mater. 121, 461 (1993).

[72] S. Hashimoto, Y. Ochiai, and K. Aso, Jpn. J. Appl. Phys. 28, 1596 (1989).

[73] S.-K. Kim and S.-C. Shin, J. Appl. Phys. 89, 3055 (2001).

[74] É. du Trémolet de Lacheisserie, D. Gignoux, and M. Schlenker (eds.), in Magnetism: Fundamentals, Magnetism (Springer, New York, 2005), Chap. 7, p. 251.

[75] B. N. Engel, C. D. England, R. A. Van Leeuwen, M. H. Wiedmann, and C. M. Falco, Phys. Rev. Lett. 67, 1910 (1991).

[76] J. Carrey, A. E. Berkowitz, W. F. Egelhoff, and D. J. Smith, Appl. Phys. Lett. 83, 5259 (2003). 
[77] H. Fujiwara, H. Kadomatsu, and T. Tokunaga, J. Magn. Magn. Mater. 31-34, 809 (1983).

[78] H. Takahashi, S. Tsunashima, S. Iwata, and S. Uchiyama, Jpn. J. Appl. Phys. 32, L1328 (1993).

[79] K. Kyuno, J.-G. Ha, R. Yamamoto, and S. Asano, Phys. Rev. B 54, 1092 (1996).

[80] K. Kyuno, R. Yamamoto, and S. Asano, J. Phys. Soc. Jpn. 61, 2099 (1992).

[81] M. Ye, H. Choi, Y. Hwang, K.-H. Yun, and Y.-C. Chung, Thin Solid Films 589, 252 (2015).

[82] A. Fert and P. M. Levy, Phys. Rev. Lett. 44, 1538 (1980).

[83] J. B. Staunton, B. L. Györffy, J. Poulter, and P. Strange, J. Phys. C 21, 1595 (1988).

[84] J. Geissler, E. Goering, M. Justen, F. Weigand, G. Schütz, J. Langer, D. Schmitz, H. Maletta, and R. Mattheis, Phys. Rev. B 65, 020405 (2001).

[85] G. G. Low and T. M. Holden, Proc. Phys. Soc. 89, 119 (1966).
[86] P. Bruno, Phys. Rev. B 49, 13231 (1994).

[87] J. Slonczewski, J. Magn. Magn. Mater. 150, 13 (1995).

[88] M. Y. Zhuravlev, E. Y. Tsymbal, and A. V. Vedyayev, Phys. Rev. Lett. 94, 026806 (2005).

[89] D. M. Edwards and A. Umerski, in Handbook of Magnetism and Advanced Magnetic Materials, edited by Kronmuller and Parkin (John Wiley \& Sons, Chichester, 2007), Vol. 1, p. 487.

[90] M. Tischer, O. Hjortstam, D. Arvanitis, J. H. Dunn, F. May, K. Baberschke, J. Trygg, J. M. Wills, B. Johansson, and O. Eriksson, Phys. Rev. Lett. 75, 1602 (1995).

[91] A. Rogalev, F. Wilhem, N. Jaouen, J. Goulon, and J.-P. Kappler, in X-ray Magnetic Circular Dichroism: Historical Perspective and Recent Highlights, Lect. Notes Phys. Vol. 697 (Springer Verlag, Berlin, Heidelberg, 2006), p. 73.

[92] J. Ododo, J. Phys. F 13, 1291 (1983).

[93] See Supplemental Material at http://link.aps.org/supplemental/ 10.1103/PhysRevB.93.174410 for Co $K$ edge XMCD calculated spectra for various bulk and NPs phases. 